Version définitive du manuscrit publié dans / Final version of the manuscript published in :

Environmental Chemistry Letters, 2012, $n^{\circ} 10(4), 325-347$

The final publication is available at www.springerlink.com: http://dx.doi.org/10.1007/s10311-012-0359-1

\title{
Insecticidal and antifungal chemicals produced by plants. A review
}

\author{
Isabelle Boulogne $e^{1,2^{*}}$, Philippe Petit ${ }^{3}$, Harry Ozier-Lafontaine ${ }^{2}$, Lucienne Desfontaines ${ }^{2}$, Gladys \\ Loranger-Merciris ${ }^{1,2}$
}

1 Université des Antilles et de la Guyane, UFR Sciences exactes et naturelles, Campus de Fouillole, F97157, Pointe-à-Pitre Cedex (Guadeloupe), France.

2 INRA, UR1321, ASTRO Agrosystèmes tropicaux, F-97170, Petit-Bourg (Guadeloupe), France.

3 UMR QUALITROP, Université des Antilles et de la Guyane, UFR Sciences exactes et naturelles, Campus de Fouillole, F-97157, Pointe-à-Pitre Cedex (Guadeloupe), France.

${ }^{*}$ Corresponding author: Isabelle BOULOGNE.

INRA, UR1321, ASTRO Agrosystèmes tropicaux, F-97170 Petit-Bourg (Guadeloupe), France. E-mail: isabelle.boulogne@univ-ag.fr or Isabelle.Boulogne@antilles.inra.fr Tel: 0590255900 (extension 5838), Fax: 0590941663.

\begin{abstract}
Leaf-cutting ants (tribe of Attini) are one of the most important pest species of agricultural and forestry productions in the New World and economic losses caused by these ants were estimated at several million dollars per year. These ants need to live in symbiosis with a basidiomycete fungus, and due to their mutualistic interaction with the symbiotic fungus, management of Attini ants may be considered at the insecticidal or at the fungicidal level, individually, or as a combination of both strategies to increase the efficiency of an integrated control. Until now, synthetic pesticides were the main control mean for these ants, but with high injurious effects on the environment. Very few studies deal with alternative methods for the control of leaf-cutting ants including the use of insecticidal and fungicidal plants extracts. There is then a need of knowledge on phytochemicals and plants that can be possibly used as general insecticides and fungicides.

Here we review chemicals of plant origin and species with insecticidal and fungicidal activities in order to establish a listing of plants and phytochemicals, which can possibly manage leafcutting ants, but also other groups of insects that utilize fungus-based agriculture or any insects or fungi pests. An exhaustive literature search of 1965 references (from 1923 to 2010) was conducted using scientific databases, chemical databases, botanical databases and books to identify published papers related to insecticidal and fungicidal chemical compounds stemmed from plant species.

The major points are the following: 1) 119 and 284 chemical compounds have been cited in the literature as to have respectively insecticidal and fungicidal activities; 2) 656 and 1064 plant species where identified as to have respectively significant insecticidal and fungicidal activities; 3 ) 3 main chemical classes were most cited for these activities: alkaloids, phenolics, and terpenoids; 4) 20 interesting chemicals with the both activities were found; and 5) 305 plant species containing these chemicals were cited.
\end{abstract}


In conclusion, the data presented in this study showed that 20 interesting chemicals (caryophyllene oxide, cinnamaldehyde, eugenol, helenalin, linalool, menthone, myristicin, pulegone, thymol, anethole, anisaldehyde, elemicin, isopimpinellin, plumbagin, podophyllotoxin, psoralen, xanthotoxin, anonaine, solamargine, and tomatine), 2 notable plant families (Lamiaceae and Apiaceae), and 17 species of these families were particularly interesting for leaf-cutting ants' pestmanagement.

\section{Key Words}

Insecticide, fungicide, plants phytochemicals, natural pesticides, Attini ants, integrated pest management, environmental chemistry, green chemistry. 


\section{Introduction}

\subsection{Consequences of synthetic chemical pesticides}

Insect pest management is nowadays a worldwide ecological challenge mainly due to environmental pollution caused by extensive use of synthetic chemical pesticides (Rattan 2010). Synthetic pesticides have been used since 1945 in order to reduce crop damages due to plant pathogens. However, the use of pesticides has some detrimental consequences on environment, such as groundwater pollution, river eutrophication, soil erosion, excessive water use, and the development of weeds and diseases resistant to chemical control (Lichtfouse et al. 2009). It also has negative impact on health with human poisonings and their related illnesses. In 1997, the United Nations Environmental Program estimated that 26 million persons suffer from pesticide poisoning each year, around 3 million persons were hospitalized due to pesticides, and about 220,000 persons died due to pesticide poisonings (Paoletti and Pimentel 2000). In the French West Indies, organochlorine insecticides that were used to control banana weevils (Cosmopolites sordidus) and leaf-cutting ants (Acromyrmex octospinosus) were responsible for long-term pollution of soils, river water, wild animals, and vegetables cultivated in polluted zones. These pesticides also were shown to cause hepatic tumors in laboratory and probably increased the risk of prostate cancer (Multigner et al. 2010).

\subsection{Major herbivores in the Neotropics: leaf-cutting ants}

Ants represent the half of the global insect biomass and leaf-cutting ants are a high percentage of the worldwide ant societies (Benckiser 2010). Leaf-cutting ants, also called 'Gardening ants' (tribe Attini, genera Acromyrmex and Atta) (photo 1), are among the most damaging invertebrates in the Neotropics (Fowler 1978). Their geographic distribution ranges from southern United States to northern Argentina, including the West Indies (Bacci et al. 2009). Because of their voraciousness and the large amount of plant material they collect, these ants cause substantial losses to agricultural and forestry productions. Some nests of the Attini tribe may contain millions of workers and consume hundreds of kilograms of leaves per year (Mikheyev 2008). Economic losses caused by these ants were estimated at several million dollars per year (Bacci et al. 2009). Consequently, they are considered as the major herbivore in the Neotropics, the main agricultural pest of the New World and the most important pest species in South America (Mikheyev 2008; Erthal et al. 2004).

Photo 1 Workers of a leaf-cutting ant, Acromyrmex octopsinosus (Reich) (left to right: minor, medium and major workers). (C) I. Boulogne

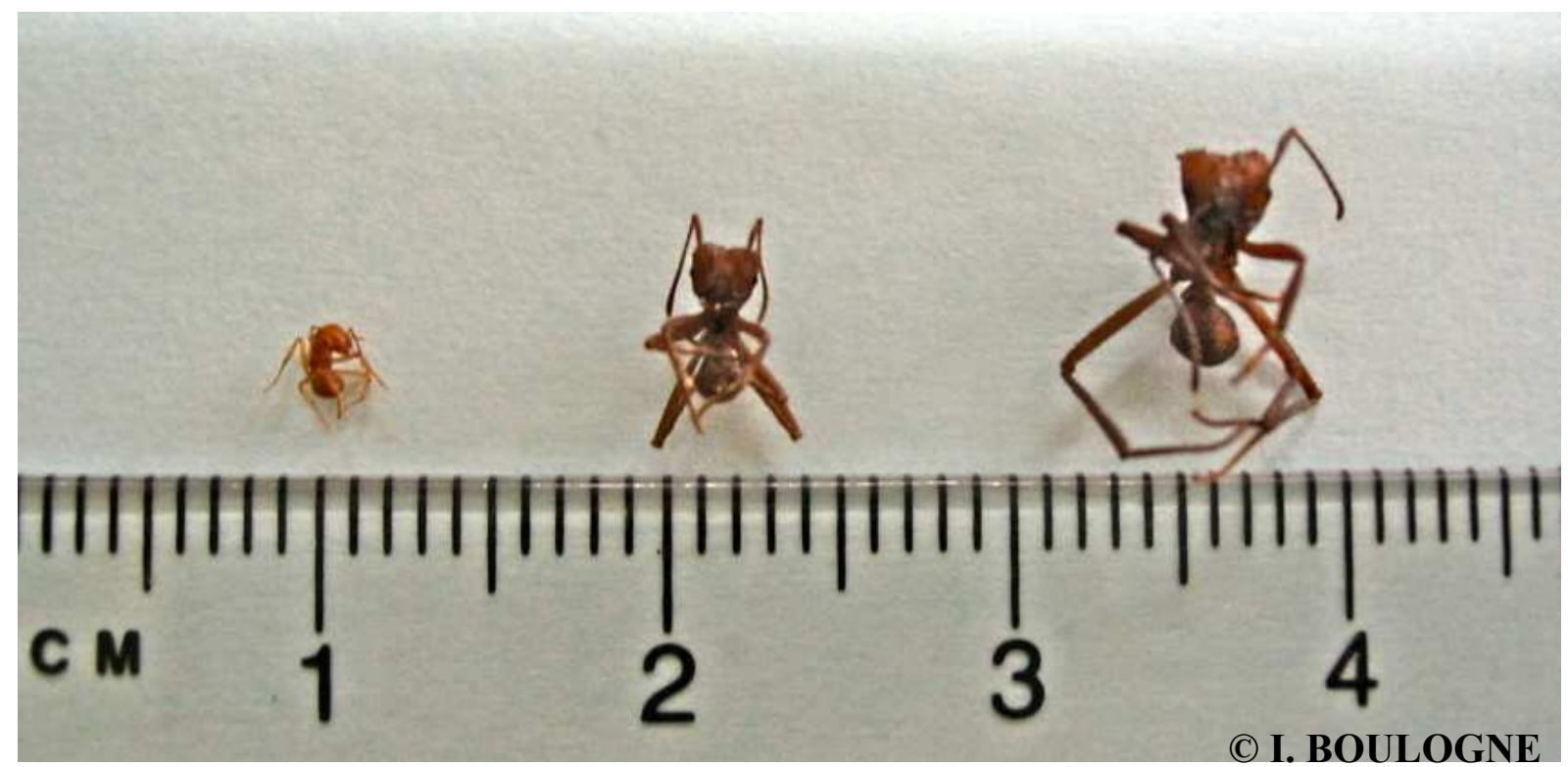


There is a binding mutualistic symbiosis between Attini ants and a Basidiomycota fungus cultivar (Mikheyev 2008). Several authors suggested that a unique fungus species (Leucocoprinus gongylophorus (Heim) Moeller) (photo 2) is cultivated by the Attini according to observations on Acromymex and Atta nests and DNA analysis studies (Silva-Pinhati et al. 2004; Silva-Pinhati et al. 2005). The fungus grows inside the nests on parts of plants (leaves, flowers,...) collected by the ants. This is an obligate relationship where the fungus metabolizes polysaccharides, such as xylan, starch, pectin, and cellulose, derived from the plants, into nutrients assimilable by the ants; in return, the ants protect the fungus from parasites and potentials competitors. The fungus is the unique food source for the ant queens, male ants, larvaes and nymphs and supplements the plant sap diet of adult workers (Silva et al. 2006). A colony of ants is composed of various castes of workers, which cooperate, in the nest, in an 'assembly line' mode. The major workers cut and bring the vegetation back to the nest. They are also known as foragers and porters. The medium-sized workers cut the plant material into smaller pieces. The smallest workers (the gardeners) implant fungal strands by applying their own fecal material on these pieces of plant material and care for the fungus as it grows. Strong relationships between ants and fungus are obvious; they are schematized in figure 1.

Photo 2 Symbiotic fungus of leaf-cutting ants, Leucocoprinus gongylophorus (Heim) Moeller. (C) I.Boulogne

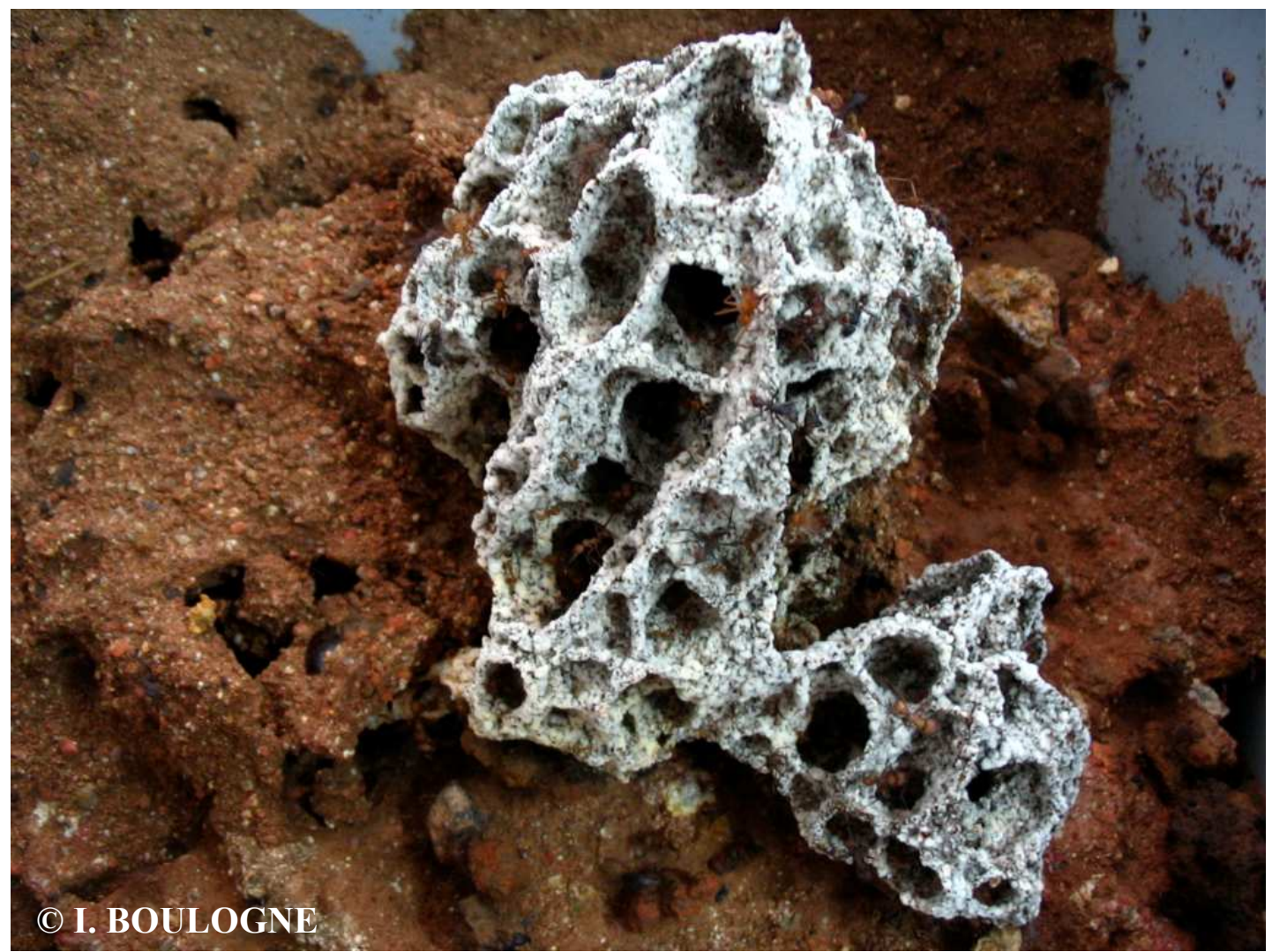

Figure 1 Relationships between ants and symbiotic fungus (Leucocoprinus gongylophorus) in leaf-cutting ants (Acromyrmex and Atta) nests. 
$\underset{3 m}{\longrightarrow}$ Foraging

$\langle\cdots \cdots \cdots \cdots\rangle \quad$ Trophallaxis

$\leftarrow-\rightarrow \rightarrow$ Symbiotic relationships

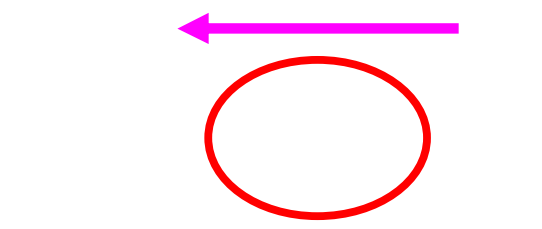

Fecundation

Levels of management

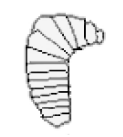

Larvaes \& Nymphs
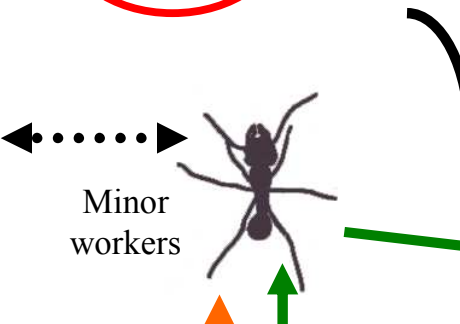

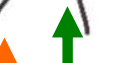

$\vdots$

Medium

workers
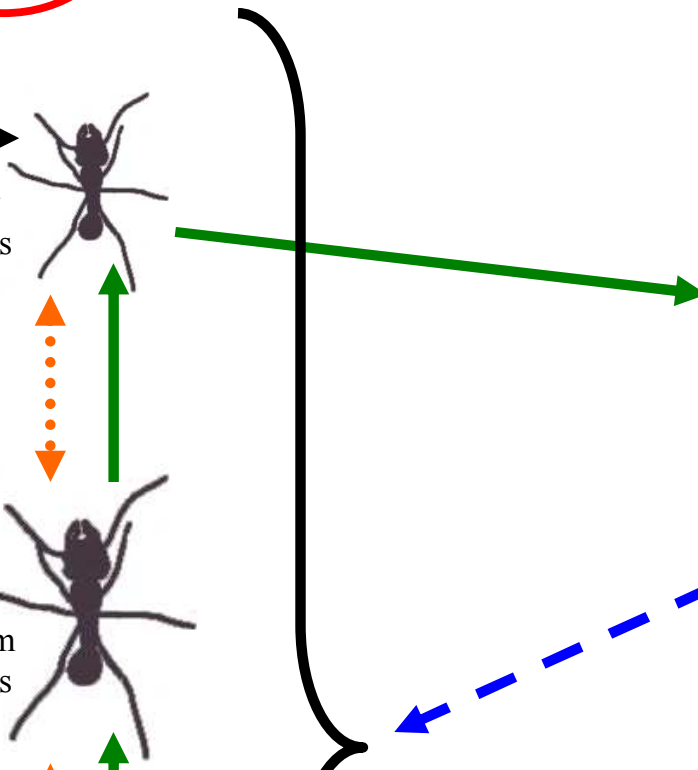

$\checkmark$

Leucocoprinus gongylophorus

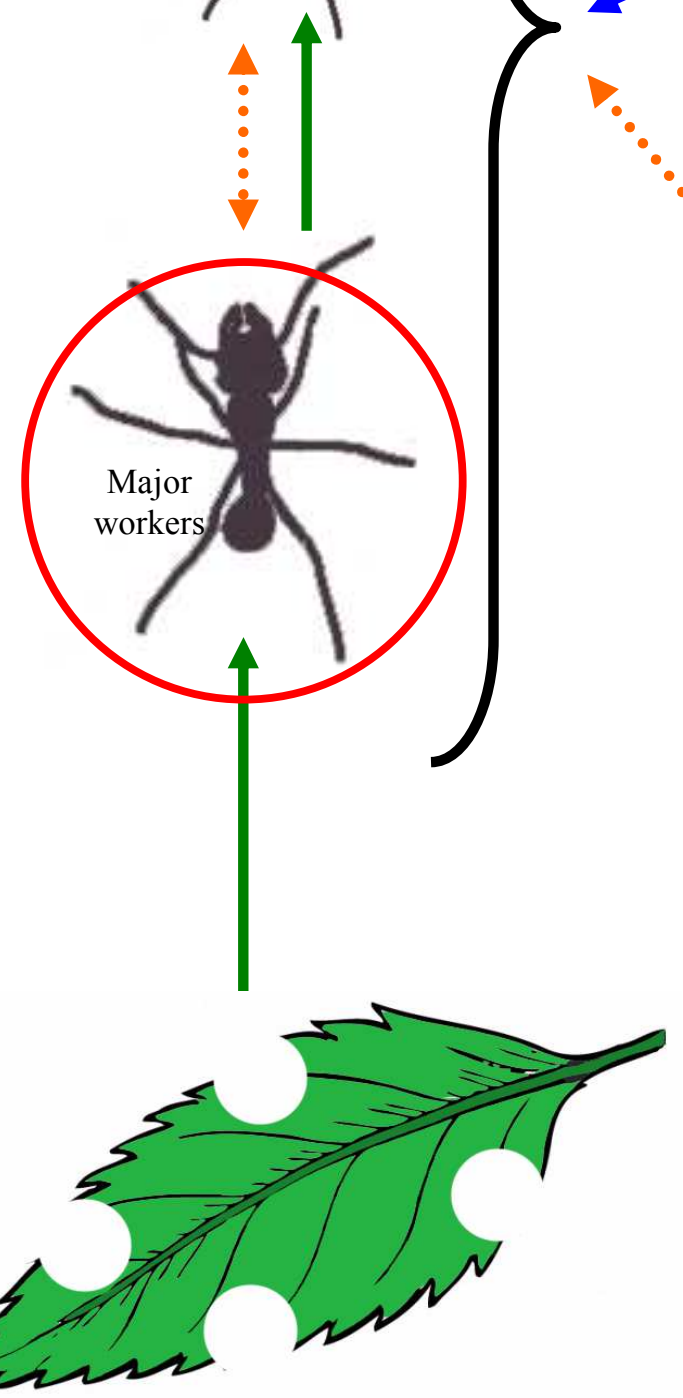

Plant material

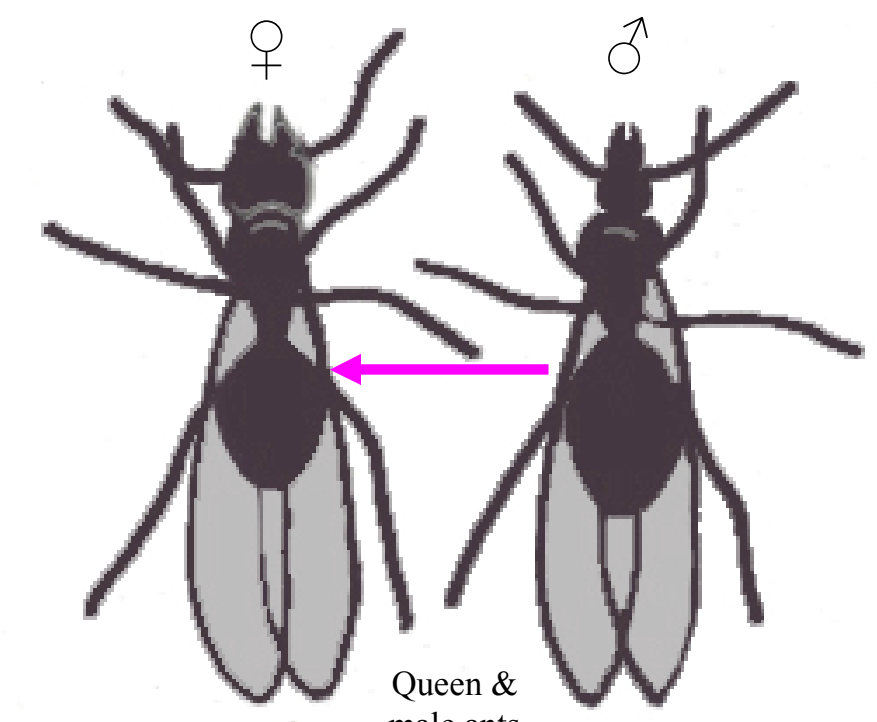


Synthetic chemical pesticides have been used worldwide, and some are still recommended, for the control of leaf-cutting ants, with no success in eradication of the pest, but causing irreversible environmental injuries (Antunes et al. 2000). Pesticides against these leaf-cutting ants have essentially focused on the use of insecticides targeted at the major ant workers. Among these pesticides, we can quote perchlordecone, sulfluramid, heptachlor, malathion, chlorpyrifos, sulfluramid and fipronil (Paoletti et al. 2000; Camargo et al. 2006; Forti et al. 2007). Some of them like sulfluramid and fipronil are still frequently and worldwide used despite of their impact on human health and environement.

\subsection{Natural pesticides}

Many plants produce biologically active metabolites. Some of which are useful as, for example, insecticide agent or insect control agent. Thus, plants constitute a vast untapped reservoir for discovering new active natural products. Applying biopesticides should decrease both pest development and the use of toxic pesticides (Lichtfouse et al. 2009). Natural products are known to be environmentally safe; they can be then a viable alternative to the use of synthetic products that can be harmful toward the environment (Regnault-Roger et al. 2003). Because of their non-phytotoxicity and systemicity as well as biodegradability, plant-derived products can be potent and valuable reagents in pest management (Javaid et al. 2006; Xuan et al. 2006; Ameziane et al. 2007). These phytochemicals are mainly biodegradable and, more importantly, they are renewable. The efficient use of such renewable natural resources is becoming increasingly an important worldwide concern (Kubo 1993) and nowadays, the green and environmental chemistry has an international success (Lichtfouse et al. 2011). Applying these biopesticides should also be an economical strategy for farmer because besides being less pollutant, they are less expensive than synthetic pesticides.

Natural insecticides and fungicides may serve as alternatives to synthetic compounds to develop safer control agents of leaf-cuting ants, as shown by some precedent studies conducted on lignans of Myristicaceae (Pagnocca et al. 1996), ricine of Ricinus communis (Bigi et al. 2004), amides of Piperaceae (Pagnocca et al. 2006; De Paula et al. 2000), rotenoïds of Lonchocarpus sp (Petit 2004) or TRAMIL's plant extracts (Boulogne 2011; Boulogne et al. 2011).

Due to their mutualistic interaction with the symbiotic fungus, management of Attini ants may be considered at the insecticidal or at the fungicidal level, individually, or as a combination of both strategies to promote a more efficient integrated control. In the literature, there is a lack of information available on molecules and plants that can be possibly used as general insecticides and fungicides. The aim of this paper was to establish a list of plants and chemicals that can possibly be used for best management of leaf-cutting ants, based on the literature available. Plant species with both insecticidal and antifungal chemicals properties were voluntary favored here. The present work, which lies within the scope of sustainable development, aim at selecting plants whose chemicals can be potentially used to control leaf-cutting ants that devastate the crops in many tropical areas. This review could also help for management of other groups of insects that utilize fungus-based culture like ambrosia beetles and termites or any insects and fungi pests. It deals with ecological pest control and biopesticides from plants origin.

An exhaustive literature search of 1965 references (from 1923 to 2010) was conducted using scientific databases (Sciencedirect, Springerlink and Wiley), chemical databases (Amicbase 2010; Duke 2010), botanical databases (Tropicos 2010) and books (Duke 1992; Bruneton 1999) to identify published papers related to insecticidal and fungicidal chemical compounds stemmed from plant species. Information was further analyzed to select plant species, plant families and chemicals worthy in pest-management of leaf-cutting ants.

\section{Insecticidal Activity}

\subsection{Chemical Compounds}


So far, 119 chemical compounds have been cited in the literature as to have an insecticidal activity. These chemicals compounds were distributed in 11 types (Figure 2). Among these 11 types, 3 were identified as holding strong insecticidal activity: the terpenoids (43 chemical compounds), the alkaloids (38 chemical compounds), and the phenolic compounds ( 21 chemical compounds) counting for 37,30 , and $20 \%$ of the chemicals cited, respectively (Table 1 ).

Figure 2 Frequency (\%) of chemical compounds types in plants with insecticidal activity (data obtain from literature search of 1965 references). Terpenoids, alkaloids and phenolic compounds were the most regularly cited as holding insecticidal activity with respectively 37,30 , and $20 \%$ of the chemicals.

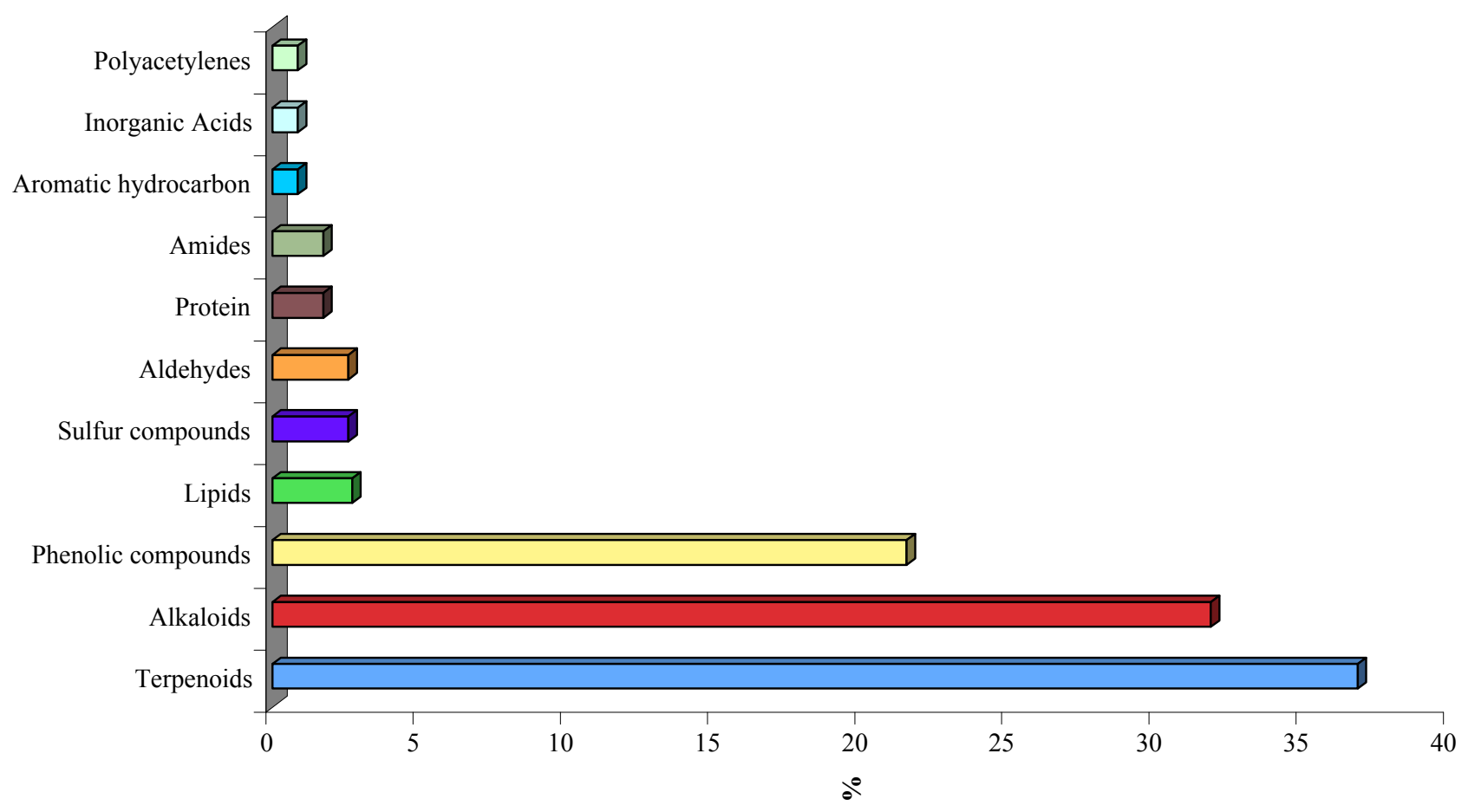

Table 1: Terpenoids, alkaloids and phenolic compounds produced by plants with insecticidal activity (data obtain from literature search of 1965 references).

\begin{tabular}{lll}
\hline Terpenoids & Alkaloids & Phenolic compounds \\
\hline$\beta$-amyrine & 5 -hydroxytryptamine & anethole \\
$\beta$-asarone & aconine & anisaldehyde \\
$\alpha$-pinene & aconitine & asarinin \\
$\alpha$-terpinene & ajaconine & bergapten \\
$\alpha$-terpineol & anabasine & canelline \\
$\alpha$-thujone & anonaine & deguelin \\
10 -hydroxy-asimicine & aphylline & deoxypodophyllotoxin \\
24 -methylene-3,22-dihydroxycholesterol & aristolochine & desoxypodophyllotoxin \\
24 -methylenecycloarta-3-ol & atropine & dillapiol \\
absinthin & benzaconine & elemicin \\
apiole & caffeine & isopimpinellin \\
ascaridol & camptothecin & mammein \\
asimicine & castanospermine & methyl-chavicol \\
azadirachtin & celabenzine & methyl-cinnamate \\
bisabolangelone & cevadine & plumbagin \\
\hline
\end{tabular}




\begin{tabular}{lll}
\hline carvone & ephedrine & podophyllotoxin \\
carvone & delcosine & sesamin \\
caryophyllene-oxide & delsoline & psoralen \\
cinerins & donaxerine & rotenone \\
cinnamaldehyde & galanthamine & rutin \\
estragole & gramine & xanthotoxin \\
eugenol & hypaconitine & \\
gedunine & jervine & \\
geranyl-linalool & mesaconitine & \\
glaucarubinone & myosmine & \\
helenalin & napelline & \\
himachalol & neoline & \\
iridomyrmecin & neopelline & \\
L-carvone & nicotine & \\
limonene & physostigmine & \\
limonene-oxide & piperine & \\
linalool & ricinine & \\
menthone & solamargine & \\
myristicin & solasonine & \\
neriifolin & sparteine & \\
ocimene & tomatine & \\
picrotoxinin & wilfordine & \\
piperitenone-oxide & wilforine & \\
pulegone & & \\
quassin & & \\
terpineol & & \\
thymol & & \\
zingiberene & & \\
\hline
\end{tabular}

\subsection{Plant Families}

Similarly, 656 plant species worldwide, distributed into 110 families, where identified as to have a significant insecticidal activity. The most cited family is the Lamiaceae, with 181 species distributed into 48 genera, counting for $28 \%$ of the plant families with an insecticidal activity (Figure 3). Within this family nine genera were the most often cited: Pycnanthemum, Teucrium, Thymus, Satureja, Micromeria, Origanum, Mentha, Monarda, and Ocimum.

Figure 3 Frequency (\%) of plant families with insecticidal activity (plants families presented have a frequency over or equal to $1 \%$ ). The most cited family is the Lamiaceae counting for $28 \%$ of the plant families cited. 


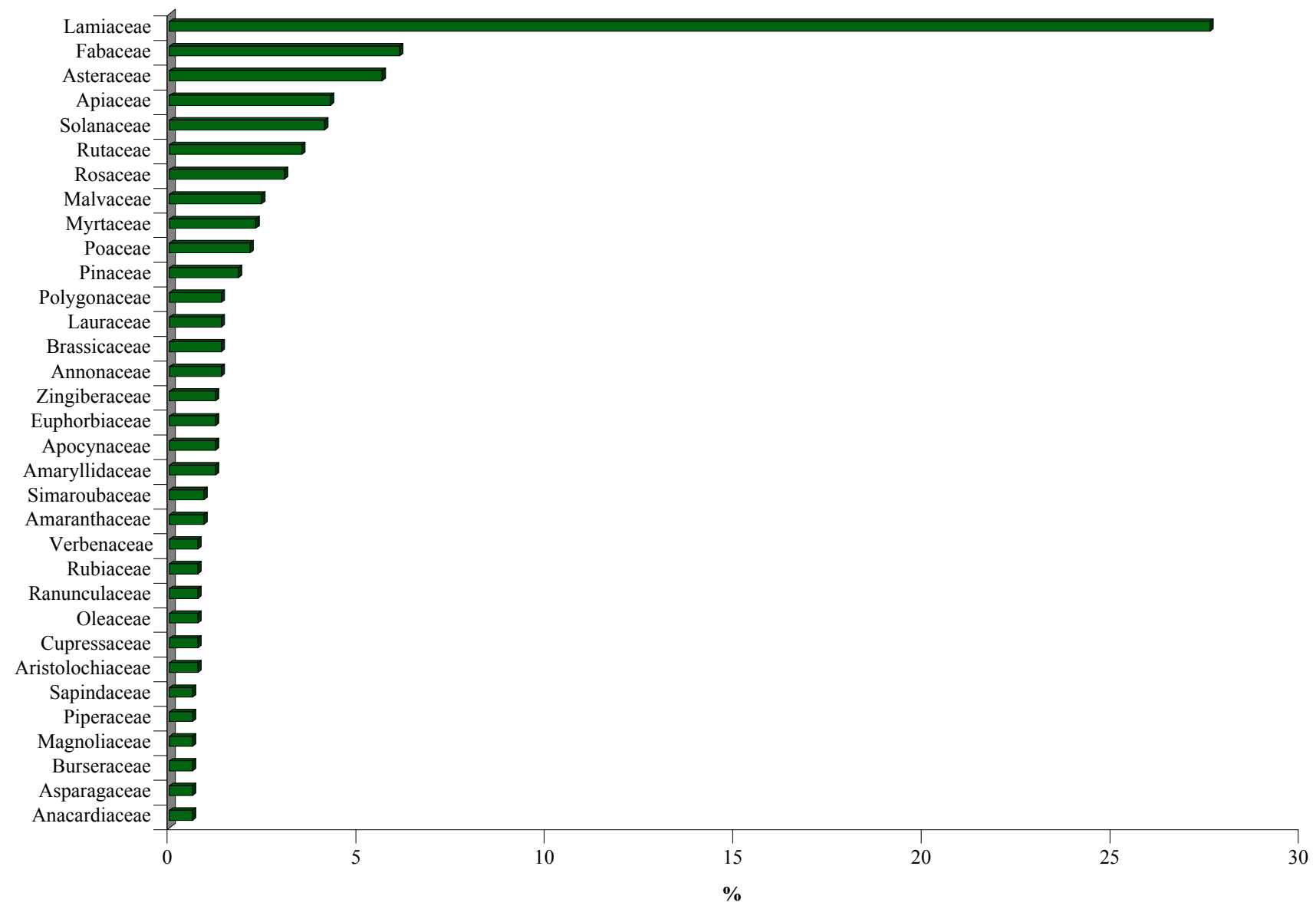

\section{Fungicidal Activity}

3.1 Chemical Compounds

From the literature, 284 chemical compounds distributed in 11 types have fungicidal activity. Of these chemical compounds types, three were the most regularly cited: the phenolic compounds ( $47 \%$ of the chemicals cited), the terpenoids ( $29 \%$ of the chemicals cited), and the alkaloids ( $11 \%$ of the chemicals cited) (Figure 4).

123 phenolic compounds, eighty terpenoids and thirty alkaloids with fungicidal activity were mentioned in the literature (Table 2).

Figure 4 Frequency (\%) of chemical compounds types with fungicidal activity (data obtain from literature search of 1965 references). Phenolic compounds (47\%), terpenoids (29\%), and the alkaloids (11\%) were the most regularly cited. 


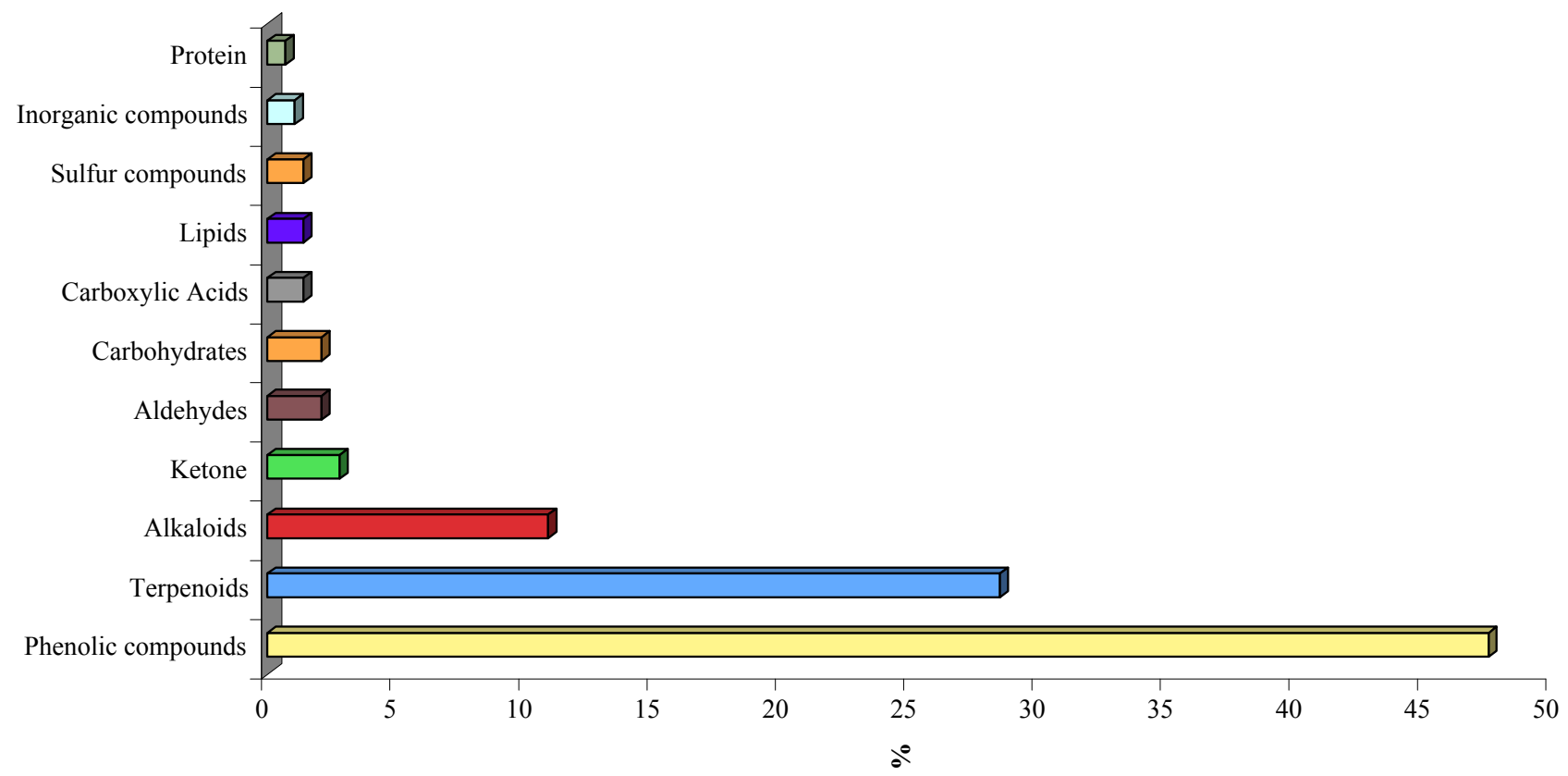

Table 2: Terpenoids, alkaloids and phenolic compounds produced by plants with fungicidal activity (data obtain from literature search of 1965 references)

\begin{tabular}{lll}
\hline \multicolumn{1}{c}{ Phenolic compounds } & \multicolumn{1}{c}{ Terpenoids } & \multicolumn{1}{c}{ Alkaloids } \\
\hline$\varepsilon$-viniferin & $\alpha$-bisabolol & $\alpha$-chaconine \\
2,6-dimethoxy-p-benzoquinone & $\alpha$-hederin & $\alpha$-solanine \\
2'-hydroxygenistein & $\beta$-ionone & 4-methoxybrassinin \\
5-methoxy-psoralen & $\alpha$-phellandrene & actinidine \\
6- $\alpha$-hydroxymaackiain & $\beta$-phellandrene & alstonine \\
6- $\alpha$-hydroxymedicarpin & 1 -tuliposide-B & amphibine \\
8-methoxy-psoralen & 1,8 -cineole & anonaine \\
aloe-emodin & 1 -tuliposide-A & arecoline \\
amentoflavone & acetophenone & berberastine \\
anacardic acid & agropyrene & berberine \\
anethole & alantolactone & canthin-6-one \\
anisaldehyde & anemonin & chelerythrine \\
baicalein & arteannuin-B & dehydroglaucine \\
benzoic-acid & asarone & dictamnine \\
biochanin-A & ascaridole & emetine \\
caffeic-acid & atractylodin & frangufoline \\
cajanin & bayogenin & isoboldine \\
catechin & borneol & jatrorrhizine \\
chavicol & butyl-phthalide & liriodenine \\
chlorogenic acid & camphor & methoxybrassitin \\
chrysarobin & capsidiol & reticuline \\
chrysin & carene & rubijervine \\
chrysophanic acid & carnosol & sanguinarine \\
chrysophanic-acid-9-anthrone & carvacrol & serpentine \\
\hline & & \\
\hline
\end{tabular}




\begin{tabular}{|c|c|c|}
\hline cinnamic acid & caryophyllene & solamargine \\
\hline cis-3,5,4'-trihydroxy-4-isopentenylstilbene & caryophyllene-oxide & solanine \\
\hline cis-resveratrol & casbene & solasodine \\
\hline coumestrol & cinnamaldehyde & theaflavin \\
\hline curcumin & cis-ocimene & \\
\hline cyclokievitone & citral & tomatine \\
\hline daidzein & citronellal & tryptanthrin \\
\hline daidzin & citronellol & \\
\hline demethylvesritol & cnidilide & \\
\hline dihydropinosylvin & coniferyl-alcohol & \\
\hline elemicin & convallamaroside & \\
\hline esculetin & cuminaldehyde & \\
\hline falcarindiol & dehydroisoeugenol & \\
\hline ferulic acid & epipolygodial & \\
\hline flavone & escin & \\
\hline formononetin & eugenol & \\
\hline furocoumarin & fulvoplumierin & \\
\hline genistein & gentiopicrin & \\
\hline genistin & geraniol & \\
\hline glyceollin-I & glutinosone & \\
\hline glycitein & gossypol & \\
\hline herniarin & hardwickic acid & \\
\hline homogentisic acid & hederagenin & \\
\hline homopisatin & hederasaponin-C & \\
\hline honokiol & helenalin & \\
\hline humulone & isoalantolactone & \\
\hline hydroxyphaseollin & kawain & \\
\hline isoliquiritin & linalool & \\
\hline isomucronulatol & medicagenic-acid & \\
\hline isopimpinellin & menthone & \\
\hline isoxanthohumol & methyl-eugenol & \\
\hline juglone & muzigadial & \\
\hline kaempferol & myrcene & \\
\hline kievitone & myristicin & \\
\hline kuwanon-G & nimbidin & \\
\hline kuwanon-H & nimbin & \\
\hline lapachol & paeonol & \\
\hline lawsone & parthenolide & \\
\hline licoisoflavone-A & patchouli-alcohol & \\
\hline liquiritigenin & p-cymene & \\
\hline liquiritin & perillaldehyde & \\
\hline lupulone & perillyl-alcohol & \\
\hline magnolol & pinene & \\
\hline mangostin & plumericine & \\
\hline medicagol & pogostone & \\
\hline medicarpin & protoanemomin & \\
\hline
\end{tabular}




\begin{tabular}{|c|c|}
\hline methyl-salicylate & pulegone \\
\hline naringenin & rishitin \\
\hline nepodin & sclareol \\
\hline nobiletin & solavetivone \\
\hline o-coumaric acid & terpinen-4-ol \\
\hline odoratol & terpinolene \\
\hline oxypeucedanin & thymol \\
\hline p-coumaric acid & tuliposide-C \\
\hline phaseol & vanillin \\
\hline phaseollidin & withaferin-A \\
\hline phaseollin & \\
\hline phenol & \\
\hline phloroglucinol & \\
\hline phylloquinone & \\
\hline piceatannol & \\
\hline piceid & \\
\hline pimpinellin & \\
\hline pinocembrin & \\
\hline pinostrobin & \\
\hline pinosylvin & \\
\hline pisatin & \\
\hline plumbagin & \\
\hline p-methoxy-cinnamic acid & \\
\hline podophyllotoxin & \\
\hline protocatechuic acid & \\
\hline prunetin & \\
\hline prunin & \\
\hline psoralen & \\
\hline psoralidin & \\
\hline pyrogallol & \\
\hline quercetin & \\
\hline resorcinol & \\
\hline resveratrol & \\
\hline rhein & \\
\hline sakuranetin & \\
\hline scopoletin & \\
\hline seselin & \\
\hline sinapic acid & \\
\hline sinensetin & \\
\hline tangeretin & \\
\hline taxifolin & \\
\hline tectorigenin & \\
\hline tetrahydroxystilbene & \\
\hline trans-3,5,4'-trihydroxy-4-isopentenylstilbene & \\
\hline trans-resveratrol & \\
\hline trichocarpin & \\
\hline
\end{tabular}




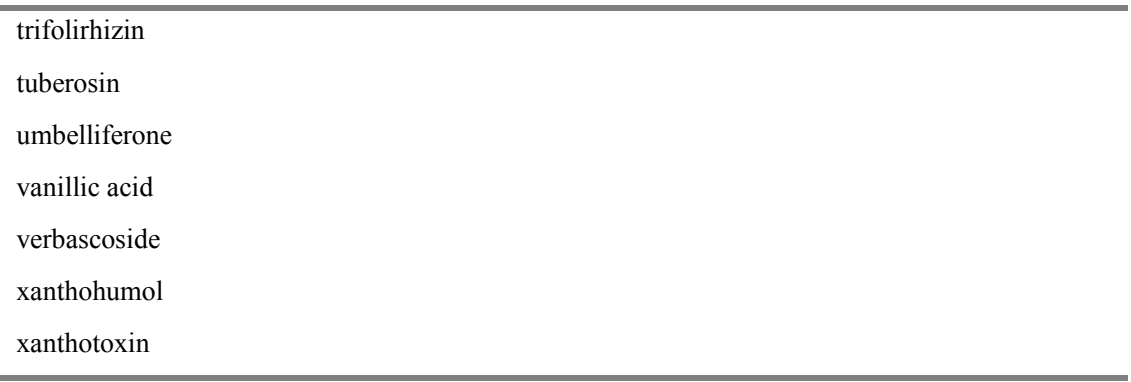

\subsection{Plant Families}

From the literature, 1064 plant species worldwide, distributed into 150 families, have fungicidal activity. The families most often cited are the Lamiaceae and the Fabaceae, representing 19 and $18 \%$ of the plant families with a fongicidal activity, respectively (Figure 5). Within the Lamiaceae, 196 species with a fungicidal activity are distributed into 48 genera. Among these genera, 9 were regularly cited: Teucrium, Pycnanthemum, Thymus, Satureja, Origanum, Micromeria, Mentha, Monarda, and Ocimum. Similarly, within the Fabaceae 190 species with a fungicidal activity are distributed into 94 genera. Among of them, 8 were most often cited: Genista, Rhynchosia, Canavalia, Trifolium, Acacia, Chamaecytisus, Cytisus, and Vigna.

Figure 5 Frequency (\%) of plant families with fungicidal activity (plants families presented have a frequency over or equal to $1 \%$ ). The families most often cited are the Lamiaceae and the Fabaceae, representing respectively 19 and $18 \%$ of the plant families.

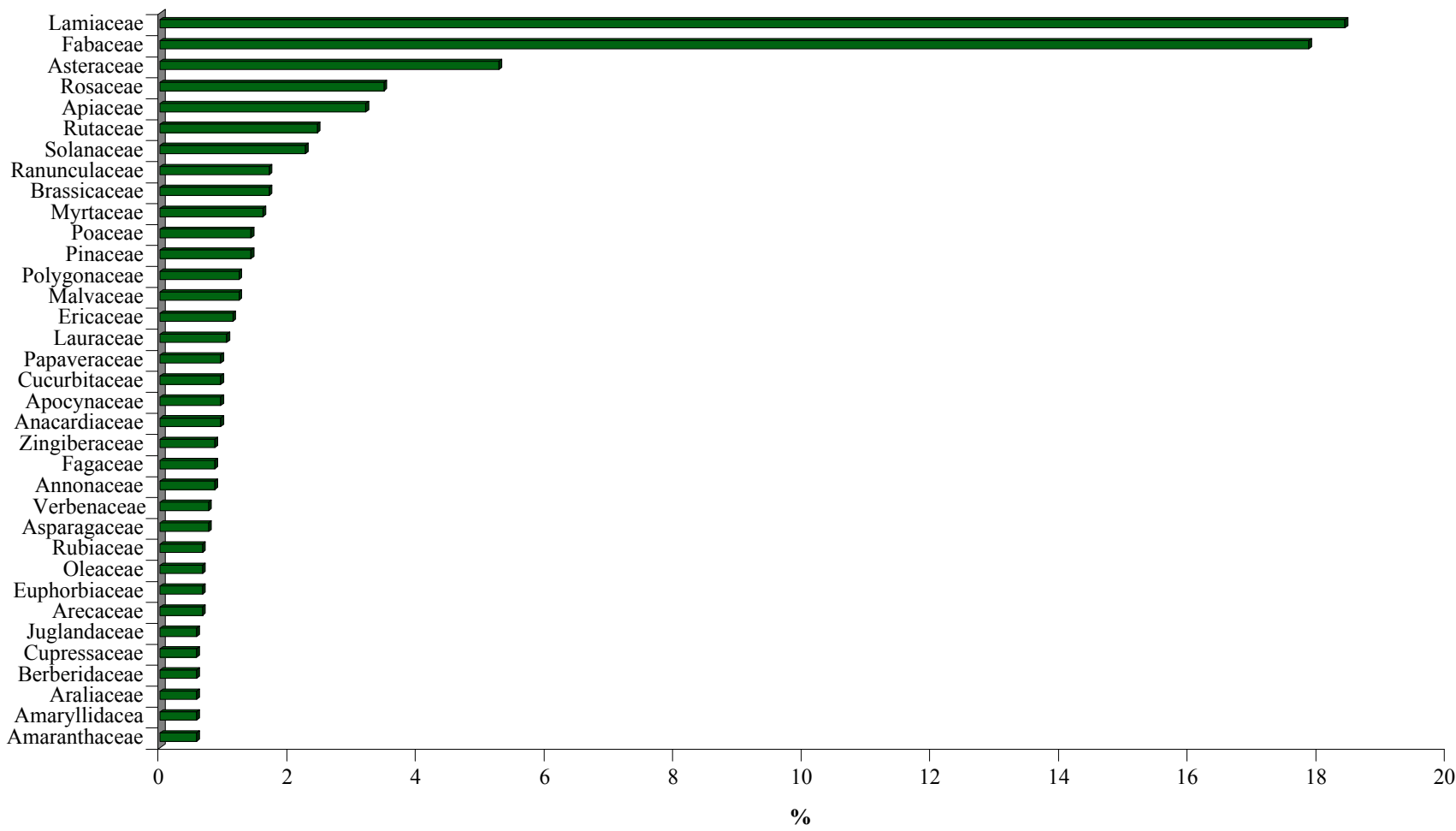

\section{Chemicals and plants species of interest for management of leaf-cutting ants}

This literature review pointed out that twenty chemical compounds are of particular interest in the perspective of management of Attini ants, as they showed both strong insecticidal and fungicidal activities. Nine of these chemical compounds are from the terpenoid family (caryophyllene oxide, cinnamaldehyde, eugenol, helenalin, linalool, menthone, myristicin, pulegone and thymol), eight from the phenolic family (anethole, anisaldehyde, elemicin, isopimpinellin, plumbagin, podophyllotoxin, 
psoralen, and xanthotoxin) and three from the alkaloid family (anonaine, solamargine, and tomatine) (Table 3, Figure 6).

Table 3: Twenty chemical compounds (phenolics, alkaloids, and terpenoids) produced by plants with both insecticidal and fungicidal activity and that have particular interest in the perspective of management of Attini ants. Some insects and fungi tested, methods of extraction and method of identification most cited were also

\begin{tabular}{|c|c|c|c|c|}
\hline Chemicals & Insects & Fungi & Extraction & Identification \\
\hline $\begin{array}{l}\text { Anethole } \\
\text { (Phenolics) }\end{array}$ & $\begin{array}{l}\text { tobacco cutworm, Spodoptera } \\
\text { litura (Akhtar and Isman, } \\
\text { 2003), Blattella germanica } \\
\text { (Chang and Ahn 2002) }\end{array}$ & $\begin{array}{l}\text { Aspergillus parasiticus (Karapinar } \\
1990 \text { ) }\end{array}$ & $\begin{array}{l}\text { hydro-distillation } \\
\text { (Osei-Safo et al. } \\
\text { 2010) }\end{array}$ & $\begin{array}{l}\text { gas chromatography- } \\
\text { mass spectrometry (GC- } \\
\text { MS) (Osei-Safo et al. } \\
\text { 2010) }\end{array}$ \\
\hline $\begin{array}{l}\text { Anisaldehyde } \\
\text { (Phenolics) }\end{array}$ & $\begin{array}{l}\text { larvae of Lycoriella ingenua } \\
\text { (Park et al. 2006) }\end{array}$ & $\begin{array}{l}\text { blue mould of pear, Penicillium } \\
\text { expansum (Neri et al. 2005) }\end{array}$ & $\begin{array}{l}\text { hydro-distillation } \\
\text { (Park et al. 2006) }\end{array}$ & $\begin{array}{l}\text { GC-MS (Park et al. } \\
\text { 2006) }\end{array}$ \\
\hline $\begin{array}{l}\text { Anonaine } \\
\text { (Alkaloids) }\end{array}$ & $\begin{array}{l}\text { black bean aphid, } \\
\text { chrysanthemum aphid and the } \\
\text { aphid Macrosiphum solanifolii } \\
\text { (Harper et al, 1947), } \\
\text { Callosobruchus chinensis } \\
\text { (Kotkar et al. 2002) }\end{array}$ & $\begin{array}{l}\text { Candida spp (Agnihotri et al. } \\
\text { 2008) }\end{array}$ & $\begin{array}{l}\text { hexane and } \mathrm{MeOH} \\
\text { and subjected to } \\
\text { column } \\
\text { chromatography } \\
\text { over silica gel } \\
\text { (Simas et al. } 2001 \text { ) }\end{array}$ & $\begin{array}{l}\text { purified by preparative } \\
\text { thin-layer } \\
\text { chromatography (TLC) } \\
\text { (Simas et al. 2001) }\end{array}$ \\
\hline $\begin{array}{l}\text { Caryophyllene } \\
\text { oxide } \\
\text { (Terpenoids) }\end{array}$ & $\begin{array}{l}\text { termite Coptotermes } \\
\text { formosanus (Cheng et al. } \\
2004 \text { ) }\end{array}$ & $\begin{array}{l}\text { white-rot fungi : Lenzites betulina, } \\
\text { Pycnoporus coccineus, Trametes } \\
\text { versicolor and Laetiporus } \\
\text { sulphureus (Cheng et al. 2004) }\end{array}$ & $\begin{array}{l}\text { hydro-distillation } \\
\text { (Cheng et al. 2004) }\end{array}$ & $\begin{array}{l}\text { GC-MS (Cheng et al. } \\
\text { 2004) }\end{array}$ \\
\hline $\begin{array}{l}\text { Cinnamaldehyde } \\
\text { (Terpenoids) }\end{array}$ & $\begin{array}{l}\text { stored product beetle, } \\
\text { Callosobruchus maculates } \\
\text { (Hubert et al. 2008; Islam et } \\
\text { al. 2009), Formosan } \\
\text { subterranean termite, } \\
\text { Coptotermes formosanus (Zhu } \\
\text { et al. 2001) }\end{array}$ & $\begin{array}{l}\text { Malassezia furfur, Candida } \\
\text { albicans (Ferhout et al. 1999) }\end{array}$ & $\begin{array}{l}\text { hydro-distillation } \\
\text { (Islam et al. 2009) }\end{array}$ & $\begin{array}{l}\text { GC-MS (Islam et al. } \\
\text { 2009) }\end{array}$ \\
\hline $\begin{array}{l}\text { Elemicin } \\
\text { (Phenolics) }\end{array}$ & $\begin{array}{l}\text { Coleopterans (Paneru et al. } \\
\text { 1997) }\end{array}$ & $\begin{array}{l}\text { yeasts, dermatophytes and } \\
\text { Aspergillus spp (Tavares et al. } \\
2008 \text { ) }\end{array}$ & $\begin{array}{l}\text { hydro-distillation } \\
\text { (Tavares et al. } \\
\text { 2008) }\end{array}$ & $\begin{array}{l}\text { GC-MS (Tavares et al. } \\
\text { 2008) }\end{array}$ \\
\hline $\begin{array}{l}\text { Eugenol } \\
\text { (Terpenoids) }\end{array}$ & $\begin{array}{l}\text { maize weevil, red flour beetle, } \\
\text { grain weevil and larger grain } \\
\text { borer (Huang et al. 2002), } \\
\text { american cockroach } \\
\text { Periplaneta americana (Ngoh } \\
\text { et al. 1998) }\end{array}$ & $\begin{array}{l}\text { wood-rot fungi (Basidiomycetes), } \\
\text { yeasts belonging to Candida } \\
\text { species (Saccharomycetes), } \\
\text { Penicillium sp (Ascomycetes) and } \\
\text { other dermatophyte fungi } \\
\text { (Vasquez et al. 2001; Gayoso et } \\
\text { al. 2005; Cheng et al. 2008; } \\
\text { Jianhua and Hai, 2001) }\end{array}$ & $\begin{array}{l}\text { methanol (Zhu et } \\
\text { al. 2001; Borg- } \\
\text { Karlson et al. } \\
\text { 2006) }\end{array}$ & $\begin{array}{l}\text { GC-MS (Zhu et al. } \\
\text { 2001; Borg-Karlson et } \\
\text { al. 2006) }\end{array}$ \\
\hline $\begin{array}{l}\text { Helenalin } \\
\text { (Terpenoids) }\end{array}$ & $\begin{array}{l}\text { vector of Chagas' disease } \\
\text { (Maya et al. 2007) }\end{array}$ & $\begin{array}{l}\text { yeasts (Ascomycetes) (Picman } \\
1984)\end{array}$ & $\begin{array}{l}\text { chloroform } \\
\text { (Staneva et al. } \\
\text { 2010) }\end{array}$ & $\begin{array}{l}\text { spectrophotometric, } \\
\text { RPLC, GC, and GC- } \\
\text { MSD and NMR } \\
\text { spectroscopy (Staneva } \\
\text { et al. 2010) }\end{array}$ \\
\hline $\begin{array}{l}\text { Isopimpinellin } \\
\text { (Phenolics) }\end{array}$ & $\begin{array}{l}\text { African Cotton Leafworm, } \\
\text { Spodoptera littoralis } \\
\text { (Hadacek et al. 1994) }\end{array}$ & $\begin{array}{l}\text { Cladosporium herbarum, Botrytis } \\
\text { cinerea, Alternaria brassicicola, } \\
\text { Dreschslera sorkiniana, } \\
\text { Alternaria } \text { spp. Bipolaris spp. and }\end{array}$ & $\begin{array}{l}\text { petroleum ether- } \\
\text { diethyl } \\
\text { ether or hexane- } \\
\text { diethyl ether- }\end{array}$ & $\begin{array}{l}\text { thin-layer } \\
\text { chromatography (TLC) } \\
\text { and high-performance } \\
\text { liquid and }\end{array}$ \\
\hline
\end{tabular}




\begin{tabular}{|c|c|c|c|c|}
\hline & & $\begin{array}{l}\text { Fusarium spp. (Al-Barwani and } \\
\text { Eltayeb 2004) }\end{array}$ & $\begin{array}{l}\text { methanol } \\
\text { (Hadacek et al. } \\
\text { 1994) }\end{array}$ & $\begin{array}{l}\text { chromatography } \\
\text { (HPLC) (Hadacek et al. } \\
\text { 1994; Al-Barwani and } \\
\text { Eltayeb 2004) }\end{array}$ \\
\hline $\begin{array}{l}\text { Linalool } \\
\text { (Terpenoids) }\end{array}$ & $\begin{array}{l}\text { adult coleopteran (Shaaya et } \\
\text { al. 1991), mediterranean fruit } \\
\text { fly (Salvatore et al. 2004), } \\
\text { mosquito Culex pipiens } \\
\text { molestus (Traboulsi et al. } \\
\text { 2002) }\end{array}$ & $\begin{array}{l}\text { several species of Aspergillus, } \\
\text { Cladosporium, Penicillium, } \\
\text { Trichoderma, Chaetomium, } \\
\text { Paecilomyces and Stachybotrys } \\
\text { (Rakotonirainy and Lavedrine } \\
\text { 2005) }\end{array}$ & $\begin{array}{l}\text { ethanol (Keszei et } \\
\text { al. 2010) }\end{array}$ & $\begin{array}{l}\text { GC-MS (Keszei et al. } \\
\text { 2010) }\end{array}$ \\
\hline $\begin{array}{l}\text { Menthone } \\
\text { (Terpenoids) }\end{array}$ & $\begin{array}{l}\text { red flour beetle, Tribolium } \\
\text { castaneum, rice weevil, } \\
\text { Sitophilus oryzae, sawtoothed } \\
\text { grain beetle, Oryzaephilus } \\
\text { surinamensis, house fly, } \\
\text { Musca domestica and German } \\
\text { cockroach, Blattella } \\
\text { germanica (Lee et al. 2003) }\end{array}$ & $\begin{array}{l}\text { Fusarium verticillioides } \\
\text { (Dambolena et al. 2008), Botrytis } \\
\text { spp., Sclerotinia spp. (Martinez- } \\
\text { Romero et al. 2008), Botrytis } \\
\text { cinerea, Fusarium solani var. } \\
\text { coeruleum and Clavibacter } \\
\text { michiganensis subsp. } \\
\text { michiganensis (Daferera et al. } \\
\text { 2003) }\end{array}$ & $\begin{array}{l}\text { hydro-distillation } \\
\text { (Daferera et al. } \\
2003 \text { ) }\end{array}$ & $\begin{array}{l}\text { GC-MS (Lee et al. } \\
\text { 2001, Daferera et al. } \\
\text { 2003) }\end{array}$ \\
\hline $\begin{array}{l}\text { Myristicin } \\
\text { (Terpenoids) }\end{array}$ & $\begin{array}{l}\text { fly, aphid, beetle, } \\
\text { and caterpillar species } \\
\text { (Dussourd 2003); coconut leaf } \\
\text { beetle, Brontispa longissima } \\
\text { (Qin et al. 2010) }\end{array}$ & $\begin{array}{l}\text { soil fungus, Gaeumannomyces } \\
\text { graminis (Monsalvez et al. 2010) }\end{array}$ & $\begin{array}{l}\text { hydro-distillation } \\
\text { (Monsalvez et al. } \\
\text { 2010; Qin et al. } \\
\text { 2010) }\end{array}$ & $\begin{array}{l}\text { GC-MS (Monsalvez et } \\
\text { al. 2010; Qin et al. } \\
\text { 2010) }\end{array}$ \\
\hline $\begin{array}{l}\text { Plumbagin } \\
\text { (Phenolics) }\end{array}$ & $\begin{array}{l}\text { subterranean termite, } \\
\text { Odontotermes obesus } \\
\text { (Ganapaty et al. 2004; Adfa et } \\
\text { al. 2010), larvae of Aedes } \\
\text { aegypti (Sreelatha et al. 2010) }\end{array}$ & $\begin{array}{l}\text { Aspergillus fumigatus, Aspergillus } \\
\text { flavus, Aspergillus niger, Candida } \\
\text { parapsilosis, Candida tropicalis, } \\
\text { C. albicans, C. neoformans, } T \text {. } \\
\text { mentagrophytes, S. schenckii, } \\
\text { Trichoderma viride, Microsporum } \\
\text { gypseum, Absidia ramosa, } \\
\text { Pseudallescheria boydii and } \\
\text { Saccharomyces (Mishra et al. } \\
\text { 2010) }\end{array}$ & $\begin{array}{l}\text { chloroform } \\
\text { (Ganapaty et al. } \\
\text { 2004), petroleum } \\
\text { ether and ethanol } \\
\text { (Mishra et al. } \\
\text { 2010) }\end{array}$ & $\begin{array}{l}\text { characterized by its EI } \\
\text { mass } \\
\text { spectra, H NMR } \\
\text { spectra, IR data, and } \\
\text { melting point and } \\
\text { identified by a search in } \\
\text { AntiBase (Ganapaty et } \\
\text { al. 2004) }\end{array}$ \\
\hline $\begin{array}{l}\text { Podophyllotoxin } \\
\text { (Phenolics) }\end{array}$ & $\begin{array}{l}\text { vinegar fly Drosophila } \\
\text { melanogaster, diamondback } \\
\text { moth Plutella xylostella and } \\
\text { common house mosquito } \\
\text { Culex pipiens pallens, } \\
\text { silkworm Bombyx mori and } \\
\text { cabbage butterfly Pieris rapea } \\
\text { (Di et al. 2010); northern } \\
\text { armyworm Mythimna } \\
\text { separata (Xu and He 2010); } \\
\text { larvae of Pieris rapae (Gao et } \\
\text { al. 2004) }\end{array}$ & $\begin{array}{l}\text { Aspergillus niger, Geotrichum } \\
\text { flavo-bnmneum, Microspotum } \\
\text { canis, Fusarium culmorum, } \\
\text { Trichophyton erinacei (Figgitt et } \\
\text { al. 1989) }\end{array}$ & $\begin{array}{l}\text { hydro-distillation } \\
\text { (Gawde et al. } \\
2009 \text { ) }\end{array}$ & $\begin{array}{l}\text { HPLC (Gawde et al. } \\
\text { 2009) }\end{array}$ \\
\hline $\begin{array}{l}\text { Psoralen } \\
\text { (Phenolics) }\end{array}$ & $\begin{array}{l}\text { beet armyworm Spodoptera } \\
\text { exigua (Diawara et al. 1993) }\end{array}$ & $\begin{array}{l}\text { Fusarium oxysporum, Fusarium } \\
\text { solani, Penicillium digitatum, } \\
\text { Penicillium italicum (Asthana et } \\
\text { al. 1993); Alternaria alternata, }\end{array}$ & $\begin{array}{l}\text { ethanol (Bafi- } \\
\text { Yeboa et al. 2005) }\end{array}$ & $\begin{array}{l}\text { HPLC (Bafi-Yeboa et } \\
\text { al. 2005) }\end{array}$ \\
\hline
\end{tabular}




\begin{tabular}{|c|c|c|c|c|}
\hline & & $\begin{array}{l}\text { Aspergillus fumigatus, } \\
\text { Microsporum gypseum, } \\
\text { Pseudallescheria boydii, Rhizopus } \\
\text { sp., Trichophyton mentagrophytes, } \\
\text { Candida albicans, Cryptococcus } \\
\text { neoformans,Wangiella } \\
\text { dermatitidis (Bafi-Yeboa et al. } \\
2005 \text { ) }\end{array}$ & & \\
\hline $\begin{array}{l}\text { Pulegone } \\
\text { (Terpenoids) }\end{array}$ & $\begin{array}{l}\text { german cockroach, house fly } \\
\text { and storage pests: rice weevil, } \\
\text { red flour beetle and } \\
\text { sawtoothed grain beetle } \\
\text { (Dancewicz et al. 2008); } \\
\text { mosquito Aedes aegypti } \\
\text { (Waliwitiya et al. 2009) }\end{array}$ & $\begin{array}{l}\text { scab of cucurbit, an Ascomycota } \\
\text { (Dancewicz et al. 2008) }\end{array}$ & $\begin{array}{l}\text { hydro-distillation } \\
\text { (Vagionas et al. } \\
\text { 2007) }\end{array}$ & $\begin{array}{l}\text { GC-MS (Vagionas et al. } \\
\text { 2007) }\end{array}$ \\
\hline $\begin{array}{l}\text { Solamargine } \\
\text { (Alkaloids) }\end{array}$ & $\begin{array}{l}\text { mosquitos (Ribeiro et al. } \\
\text { 2009); potato aphid, } \\
\text { Macrosiphum euphorbiae } \\
\text { (Guntner et al. 2000) }\end{array}$ & $\begin{array}{l}\text { Ascomycetes plant pathogenic } \\
\text { fungi (Fewell et al. 1994) }\end{array}$ & $\begin{array}{l}\text { methanol and } \\
\text { purified by } \\
\text { different } \\
\text { procedures (Vencl } \\
\text { et al. 1999) }\end{array}$ & $\begin{array}{l}\text { purity was verified by } \\
\text { high-performance liquid } \\
\text { chromatography } \\
\text { (HPLC) (Hall et al. } \\
\text { 2006) }\end{array}$ \\
\hline $\begin{array}{l}\text { Tomatine } \\
\text { (Alkaloids) }\end{array}$ & $\begin{array}{l}\text { potato aphid, Macrosiphum } \\
\text { euphorbiae (Guntner et al. } \\
\text { 2000); Colorado potato beetle, } \\
\text { Leptinotarsa decemlineata } \\
\text { (Mitchell and Harrison 1985); } \\
\text { two-striped grasshopper } \\
\text { Melanopus bivittatus, tomato } \\
\text { fruit-worm Heliothis zea, } \\
\text { ichneumonid wasp Hyposoter } \\
\text { exiguae, beet armyworm } \\
\text { Spodoptera exigua (Bloem et } \\
\text { al. 1989); red flour beetle, } \\
\text { Tribolium castaneum, rice } \\
\text { weevil, Sitophilus oryzae } \\
\text { (Nenaah 2010) }\end{array}$ & $\begin{array}{l}\text { Fusarium solani, Nomuraea rileyi } \\
\text { (Gallardo et al. 1990); Fusarium } \\
\text { oxysporum (Ito et al. 2007) }\end{array}$ & $\begin{array}{l}\text { ethanol (Nenaah } \\
\text { 2010) }\end{array}$ & $\begin{array}{l}\text { TLC, NMR and MS } \\
\text { (Nenaah 2010) }\end{array}$ \\
\hline $\begin{array}{l}\text { Thymol } \\
\text { (Terpenoids) }\end{array}$ & $\begin{array}{l}\text { house fly, red flour beetle, } \\
\text { southern corn rootworm, } \\
\text { American cockroach and } \\
\text { Formosan subterranean } \\
\text { termite (Zhu et al. 2003); } \\
\text { mosquito Culex pipiens } \\
\text { molestus (Traboulsi et al. } \\
\text { 2002) }\end{array}$ & $\begin{array}{l}17 \text { phytopathogenic fungi (Kordali } \\
\text { et al. 2008), Penicillium sp } \\
\text { (Vasquez et al. 2001) and } \\
\text { Candida sp (Braga et al. 2008) }\end{array}$ & $\begin{array}{l}\text { hydro-distillation } \\
\text { (Vokou et al. } \\
\text { 1998) }\end{array}$ & $\begin{array}{l}\text { GC-MS (Vokou et al. } \\
\text { 1998) }\end{array}$ \\
\hline $\begin{array}{l}\text { Xanthotoxin } \\
\text { (Phenolics) }\end{array}$ & $\begin{array}{l}\text { Spodoptera litura, S. exigua, } \\
\text { Trichoplusia ni (Akhtar and } \\
\text { Isman 2004) }\end{array}$ & $\begin{array}{l}\text { Cladosporium herbarum, Botrytis } \\
\text { cinerea, Alternaria brassicicola, } \\
\text { Dreschslera sorkiniana, Fusarium } \\
\text { spp. and Alternaria spp. (Al- } \\
\text { Barwani and Eltayeb 2004) }\end{array}$ & $\begin{array}{l}\text { petroleum ether- } \\
\text { diethyl ether or } \\
\text { hexane-diethyl } \\
\text { ether-methanol } \\
\text { (Hadacek et al. } \\
\text { 1994) }\end{array}$ & $\begin{array}{l}\text { HPLC (Hadacek et al. } \\
\text { 1994) }\end{array}$ \\
\hline
\end{tabular}


Figure 6 Twenty chemical compounds produced by plants with insecticidal and fungicidal activities that have particular interest in the perspective of management of Attini ants: anethole (a), anisaldehyde (b), anonaine (c), caryophyllene oxide (d), cinnamaldehyde (e), elemicin (f), eugenol (g), helenalin (h), isopimpinellin (i), linalool (j), menthone (k), myristicin (l), plumbagin (m), podophyllotoxin (n), psoralen (o), pulegone (p), solamargine $(\mathrm{q})$, tomatine (r), thymol (s) and xanthotoxin (t).

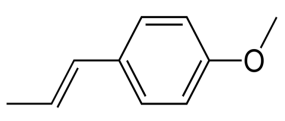

a<smiles>O=C/C=C/c1ccccc1</smiles>

e<smiles>COc1c2ccoc2c(OC)c2oc(=O)ccc12</smiles>

i<smiles>COc1ccc(C=O)cc1</smiles>

b<smiles>C=CCc1cc(OC)c(OC)c(OC)c1</smiles>

f<smiles>C=CC(C)(O)CCC=C(C)C</smiles>

j<smiles></smiles><smiles>C=C1CCCOC(C)CCC2CC(C)(C)C12</smiles>

d<smiles>C=CCc1ccc(O)c(OC)c1</smiles>

g<smiles>CC1CCC(C(C)C)C(=O)C1</smiles>

k<smiles>C=C1C(=O)OC2C[C@H](C)[C@H]3C=CC(=O)[C@@]3(O)[C@H](O)C12</smiles>

h<smiles>C=CCc1cc(OC)c2c(c1)OCO2</smiles>

I<smiles>CC1=CC(=O)c2c(O)cccc2C1=O</smiles>

m<smiles></smiles>

n<smiles>O=c1ccc2cc3ccoc3cc2o1</smiles>

o<smiles>CC(C)=C1CC[C@@H](C)CC1=O</smiles>

p
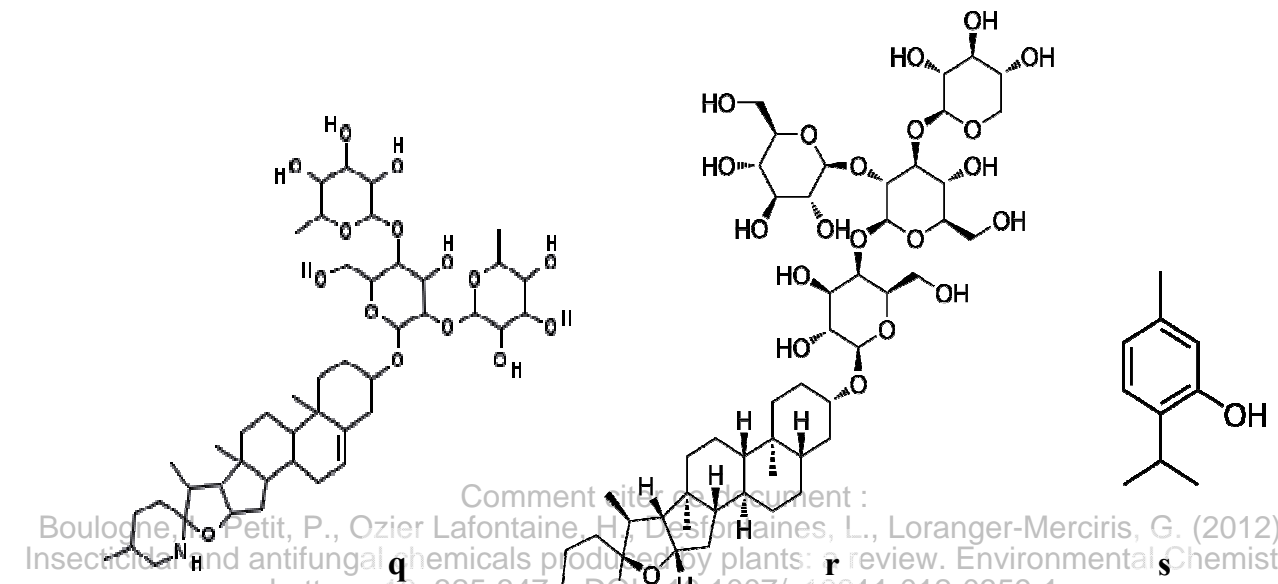<smiles>COc1c2occc2cc2ccc(=O)oc12</smiles> 
Because of the co-dependence of leaf-cutting ants and its basidiomycete fungus, it was important to our study to find interesting plant species, which contained chemicals with the both activities. Our literature search displayed 305 worldwide plant species, which contained chemicals with both insecticidal and fungicidal activity; among these plant species, 148 species belong to the Lamiaceae family and 24 species belong to the Apiaceae family. Twenty-one plant species contained from four to seven chemicals compounds with both insecticidal and fungicidal activities. Eleven of these twenty-one species belong to the family of Lamiaceae and six belong to the family of Apiaceae (Table 4).

Table 4 Plant families, plant species and their chemical compounds with both insecticidal and fungicidal activity. In bold, plant wich contained from 4 to 7 chemical compounds with both insecticidal and fungicidal activities

\begin{tabular}{|c|c|c|}
\hline Plant Families & Plant species & Chemicals \\
\hline Acoraceae & Acorus calamus L. & eugenol, elemicin, menthone \\
\hline Amaranthaceae & Chenopodium album $\mathrm{L}$. & xanthotoxin \\
\hline \multirow[t]{2}{*}{ Amaryllidaceae } & Narcissus tazetta L. & eugenol \\
\hline & Polianthes tuberosa $\mathrm{L}$. & eugenol \\
\hline \multirow[t]{7}{*}{ Annonaceae } & Annona cherimola Mill. & anonaine \\
\hline & Annona glabra $\mathrm{L}$. & anonaine \\
\hline & Annona montana $\mathrm{L}$. & anonaine \\
\hline & Annona reticulata $\mathrm{L}$. & anonaine \\
\hline & Annona squamosa $\mathrm{L}$. & anonaine \\
\hline & Cananga odorata (Lam.) Hook. f. \& Thomson & eugenol \\
\hline & Rollinia mucosa (Jacq.) Baill. & anonaine \\
\hline \multirow[t]{26}{*}{ Apiaceae } & Ammi majus L. & xanthotoxin, isopimpinellin \\
\hline & Ammi visnaga (L.) Lam. & xanthotoxin, isopimpinellin \\
\hline & Anethum graveolens $\mathrm{L}$. & eugenol, elemicin, myristicin, anethole \\
\hline & Angelica archangelica $\mathrm{L}$. & xanthotoxin, psoralen, isopimpinellin \\
\hline & Angelica dahurica Benth \& Hook. & xanthotoxin, psoralen \\
\hline & & eugenol, thymol, menthone, myristicin, \\
\hline & Apium graveolens $\mathbf{L}$. & xanthotoxin, psoralen, isopimpinellin \\
\hline & Carum carvi $\mathrm{L}$. & myristicin \\
\hline & Coriandrum sativum $\mathrm{L}$. & myristicin, psoralen \\
\hline & Cuminum cyminum $\mathrm{L}$. & eugenol, caryophyllene oxide, anisaldehyde \\
\hline & & eugenol, elemicin, caryophyllene oxide, \\
\hline & & myristicin, isopimpinellin, psoralen, \\
\hline & Daucus carota $\mathbf{L}$. & xanthotoxin \\
\hline & Ferula alliacea Boiss. & isopimpinellin \\
\hline & Ferula assa-foetida $\mathrm{L}$. & isopimpinellin \\
\hline & & myristicin, anisaldehyde, isopimpinellin, \\
\hline & Foeniculum vulgare Miller & psoralen, xanthotoxin \\
\hline & Glehnia littoralis F. Schmidt \& Miquel & psoralen, xanthotoxin \\
\hline & Heracleum laciniatum & isopimpinellin \\
\hline & Heracleum lanatum Michx. & isopimpinellin \\
\hline & Levisticum officinale W.D.J. Koch & eugenol, myristicin, psoralen \\
\hline & Oenanthe aquatica (L.) Poir. & myristicin \\
\hline & Oenanthe crocata $\mathrm{L}$. & myristicin \\
\hline & Oenanthe javanica (Blume) DC. & eugenol, myristicin \\
\hline & & myristicin, isopimpinellin, psoralen, \\
\hline & Pastinaca sativa $\mathbf{L}$. & xanthotoxin \\
\hline
\end{tabular}




\begin{tabular}{|c|c|c|}
\hline & Petroselinum crispum (Mill.) Fuss & $\begin{array}{l}\text { elemicin, myristicin, isopimpinellin, } \\
\text { psoralen, xanthotoxin }\end{array}$ \\
\hline & Pimpinella anisum $\mathrm{L}$. & eugenol, myristicin, anisaldehyde \\
\hline & Trachyspermum ammi (L.) Sprague & thymol \\
\hline \multirow[t]{2}{*}{ Aristolochiaceae } & Asarum canadense $\mathrm{L}$. & elemicin \\
\hline & Asiasarum heterotropoides (F. Schmidt) F. Maek. & elemicin \\
\hline Asparagaceae & Hyacinthus orientalis $\mathrm{L}$. & eugenol, cinnamaldehyde \\
\hline \multirow[t]{14}{*}{ Asteraceae } & Achillea millefolium $\mathrm{L}$. & eugenol \\
\hline & Ageratum conyzoides $\mathrm{L}$. & eugenol, caryophyllene oxide \\
\hline & Arnica montana $\mathrm{L}$. & helenalin, thymol \\
\hline & Artemisia аппиа $\mathrm{L}$. & caryophyllene oxide \\
\hline & Artemisia capillaris Thunb. & eugenol \\
\hline & Artemisia dracunculus $\mathbf{L}$. & eugenol, menthone, anethole, anisaldehyde \\
\hline & Artemisia herba-alba Asso. & thymol \\
\hline & Cnicus benedictus L. & cinnamaldehyde \\
\hline & Cynara cardunculus subsp. cardunculus L. & eugenol \\
\hline & Eupatorium perfoliatum $\mathrm{L}$. & helenalin \\
\hline & Helianthus annuus $\mathrm{L}$. & eugenol \\
\hline & Helichrysum angustifolium DC. & eugenol \\
\hline & Inula helenium $\mathrm{L}$. & helenalin \\
\hline & Saussurea lappa C. B. Clarke & caryophyllene oxide \\
\hline \multirow[t]{3}{*}{ Berberidaceae } & Podophyllum hexandrum Royle & podophyllotoxin \\
\hline & Podophyllum peltatum $\mathrm{L}$. & podophyllotoxin \\
\hline & Podophyllum pleianthum L. & podophyllotoxin \\
\hline \multirow[t]{3}{*}{ Burseraceae } & Boswellia sacra Flueck & anisaldehyde \\
\hline & Canarium indicum $\mathrm{L}$. & elemicin, anethole \\
\hline & Commiphora myrrha (T. Nees) Engl. & eugenol, cinnamaldehyde \\
\hline Cannabaceae & Humulus lupulus L. & eugenol, caryophyllene oxide \\
\hline \multirow[t]{2}{*}{ Cistaceae } & Cistus ladaniferus L. & eugenol \\
\hline & Juniperus communis L. & caryophyllene oxide \\
\hline Cucurbitaceae & Citrullus colocynthis (L.) Schrad. & anethole, anisaldehyde \\
\hline \multirow[t]{2}{*}{ Cupressaceae } & Juniperus sabina L. & podophyllotoxin \\
\hline & Juniperus virginiana $\mathrm{L}$. & elemicin, podophyllotoxin \\
\hline Droseraceae & Dionaea muscipula $\mathrm{E}$. & plumbagin \\
\hline \multirow[t]{2}{*}{ Ericaceae } & Vaccinium corymbosum $\mathrm{L}$. & eugenol, pulegone, thymol, myristicin \\
\hline & Vaccinium macrocarpon Aiton & eugenol, anisaldehyde \\
\hline Euphorbiaceae & Croton eluteria (L.) W. Wright & eugenol \\
\hline \multirow[t]{7}{*}{ Fabaceae } & Acacia farnesiana (L.) Willd. & eugenol, anisaldehyde \\
\hline & Copaifera spp & caryophyllene oxide \\
\hline & Glycyrrhiza glabra $\mathrm{L}$. & eugenol, thymol, anethole, xanthotoxin \\
\hline & Myroxylon balsamum (L.) Harms & eugenol, cinnamaldehyde \\
\hline & Psoralea corylifolia $\mathrm{L}$. & psoralen \\
\hline & Tamarindus indica $\mathrm{L}$. & cinnamaldehyde \\
\hline & Trifolium pratense $\mathrm{L}$. & eugenol \\
\hline Geraniaceae & Pelargonium graveolens L'Hér. & eugenol, menthone \\
\hline Ginkgoaceae & Ginkgo biloba $\mathrm{L}$. & thymol \\
\hline Iridaceae & Iris $x$ germanica $\mathrm{L}$. & eugenol \\
\hline Juglandaceae & Juglans regia $\mathrm{L}$. & eugenol \\
\hline \multirow[t]{2}{*}{ Lamiaceae } & Acinos alpinus var. meridionalis (Nyman) P. W. Ball & caryophyllene oxide \\
\hline & Acinos suaveolens $\mathrm{G}$. Don & pulegone, thymol, menthone \\
\hline
\end{tabular}


Agastache foeniculum (Pursh) Kuntze

Agastache pallidiflora (A. Heller) Rydb.

Agastache rugosa (Fischer \& C. Meyer) Kuntze

Agastache urticifolia (Benth.) Kuntze

Calamintha nepeta (L.) Savi

Calamintha nepeta subsp. glandulosa (Req.) P.W. Ball

Collinsonia canadensis $\mathrm{L}$.

Coridothymus capitatus (L.) Rchb. f.

Cunila origanoides (L.) Britton

Dracocephalum thymiflorum L.

Elsholtzia blanda (Benth.) Benth.

Elsholtzia eriostachya var. pusilla

Elsholtzia pilosa (Benth.) Benth.

Elsholtzia polystachya Benth.

Galeopsis tetrahit L.

Hedeoma drummondii Benth.

Hedeoma pulegioides (L.) Pers.

Hyptis suaveolens (L.) Poit.

Hyptis verticillata Jacq.

Hyssopus officinalis $\mathrm{L}$.

Isanthus brachiatus (L.) Bsp

Lavandula angustifolia Miller

Lavandula latifolia Medik.

Lavandula $x$ intermedia Emeric ex Loisel.

Leonotis leonurus (L.) R. Br.

Lepechinia calycina Epling

Lycopus europaeus L.

Lycopus virginicus $\mathrm{L}$.

Melissa officinalis $\mathrm{L}$.

Mentha aquatica $\mathrm{L}$.

Mentha arvensis var. piperascens Malinv. ex Holmes

Mentha longifolia (L.) Huds.

Mentha pulegium $\mathbf{L}$.

Mentha spicata $\mathrm{L}$.

\section{Mentha $x$ piperita $\mathrm{L}$.}

Mentha $x$ rotundifolia (L.) Huds.

Micromeria congesta Boiss. \& Hausskn.

Micromeria croatica (Pers.) Schott

Micromeria dalmatica Benth.

Micromeria fruticosa Druce

Micromeria fruticosa subsp. barbata (Boiss. \& Kotschy) P.H. Davis

Micromeria juliana (L.) Bentham ex Reichb.

Micromeria myrtifolia Boiss. \& Hohen.

Micromeria thymifolia Fritsch

Minthostachys mollis (Kunth) Griseb. anisaldehyde

pulegone, menthone

anisaldehyde

pulegone, menthone

pulegone, menthone

eugenol, pulegone, thymol, menthone

elemicin

thymol

thymol

pulegone, caryophyllene oxide, menthone

eugenol

caryophyllene oxide

thymol

thymol

caryophyllene oxide

pulegone, menthone

pulegone, menthone

thymol, caryophyllene oxide

podophyllotoxin

eugenol, thymol

caryophyllene oxide

caryophyllene oxide, cinnamaldehyde

eugenol, caryophyllene oxide,

cinnamaldehyde

eugenol, caryophyllene oxide,

cinnamaldehyde

caryophyllene oxide

menthone

caryophyllene oxide

pulegone

thymol, caryophyllene oxide

pulegone, caryophyllene oxide, menthone

eugenol, pulegone, menthone, anisaldehyde pulegone, thymol, caryophyllene oxide, menthone

eugenol, pulegone, thymol, menthone eugenol, pulegone, thymol, menthone eugenol, pulegone, thymol, caryophyllene oxide, menthone, anethole

pulegone, menthone

eugenol, pulegone, thymol, menthone pulegone, caryophyllene oxide, menthone pulegone

pulegone, menthone

eugenol, pulegone, thymol, caryophyllene oxide, menthone

pulegone, thymol, menthone

eugenol, pulegone, thymol, menthone

pulegone, caryophyllene oxide, menthone

pulegone, menthone 
Moldavica thymiflora (L.) Rydb.

Monarda citriodora Cerv. ex Lag.

Monarda clinopodia $\mathrm{L}$.

Monarda didyma L.

Monarda fistulosa $\mathrm{L}$

Monarda media Willd.

Monarda punctata L.

Monarda russeliana Nutt. ex Sims

Nepeta cataria L.

Nepeta racemosa Lam.

\section{Ocimum basilicum $\mathbf{L}$}

Ocimum canum Sims

Ocimum gratissimum $\mathrm{L}$.

Ocimum kilimandscharicum Baker ex Gürke

Ocimum sanctum $\mathrm{L}$.

Ocimum suave Willd.

Ocimum tenuiflorum $\mathrm{L}$.

Origanum majorana L.

Origanum minutiflorum O. Schwarz \& P.H. Davis

Origanum onites L.

Origanum sipyleum $\mathrm{L}$.

Origanum syriacum L.

Origanum vulgare L.

Origanum vulgare subsp. hirtum Ietsw.

Origanum vulgare var. hirtum (Schur) Soó

Origanum vulgare var. viride Boiss.

Perilla frutescens (L.) Britton

Pogostemon cablin (Blanco) Benth.

Pycnanthemum albescens Torr. \& A. Gray

Pycnanthemum beadlei (Small) Fernald

Pycnanthemum californicum Torr. ex Durand

Pycnanthemum clinopodioides Torr. \& A. Gray

Pycnanthemum incanum (L.) Michx.

Pycnanthemum loomisii Nutt.

Pycnanthemum montanum Michx.

Pycnanthemum muticum (Michx.) Pers.

Pycnanthemum nudum Nutt.

Pycnanthemum pilosum Nutt.

Pycnanthemum pycnanthemoides (Leavenw.) Fernald

Pycnanthemum setosum Nutt.

Pycnanthemum tenuifolium Schrad.

Pycnanthemum torreyi Benth.

Pycnanthemum verticillatum (Michx.) Pers.

Pycnanthemum virginianum (L.) Durand \& Jackson

Rosmarinus eriocalyx Jordan \& Fourr.

Rosmarinus officinalis L.

Rosmarinus tomentosus Huber

Rosmarinus x lavandulaceus De Noe pulegone, caryophyllene oxide, menthone

thymol

pulegone

pulegone, thymol

pulegone, thymol

thymol

pulegone, thymol

pulegone, thymol

pulegone, thymol, caryophyllene oxide

pulegone, thymol

eugenol, thymol, caryophyllene oxide, menthone

eugenol

eugenol, thymol

eugenol

eugenol

eugenol

caryophyllene oxide

eugenol

eugenol, thymol

eugenol, thymol, caryophyllene oxide

linalool, thymol

thymol, caryophyllene oxide

eugenol, thymol, caryophyllene oxide

thymol

thymol

thymol

elemicin, myristicin

eugenol, caryophyllene oxide,

cinnamaldehyde

pulegone, menthone

pulegone, menthone

pulegone, menthone

pulegone, menthone

pulegone, menthone

pulegone, caryophyllene oxide

pulegone, menthone

pulegone, menthone

thymol

pulegone, menthone, anisaldehyde

pulegone, menthone

eugenol, pulegone, menthone

pulegone, caryophyllene oxide, menthone

pulegone, menthone

pulegone, menthone

pulegone, thymol, menthone

caryophyllene oxide

thymol, caryophyllene oxide

caryophyllene oxide

caryophyllene oxide 
Rosmarinus x mendizabalii Sagredo Ex Rosua

Salvia canariensis L.

Salvia dorisiana Standl.

Salvia gilliesii Benth.

Salvia officinalis L.

Salvia sclarea $\mathrm{L}$

Satureja cilicica P.H. Davis

Satureja cuneifolia Ten.

Satureja douglasii (Benth.) Briq.

Satureja glabella (Michx.) Briq.

Satureja grandiflora (L.) Scheele

Satureja hortensis L.

Satureja montana L.

Satureja obovata Lag.

Satureja odora (Griseb.) Epling

Satureja parvifolia (Phil.) Epling

Satureja subspicata subsp. liburnica Silic

Satureja thymbra L.

Scutellaria churchilliana Fernald

Scutellaria galericulata L.

Scutellaria parvula Michx.

Sideritis athoa Papanicolaou \& Kokkini

Sideritis scardica Griseb.

Teucrium arduini $\mathrm{L}$.

Teucrium asiaticum $\mathrm{L}$.

Teucrium cyprium Boiss.

Teucrium divaricatum var. canescens (Čelak.) Holmboe

Teucrium gnaphalodes L'Hér.

Teucrium kotschyanum Poech

Teucrium micropodioides Rouy

Teucrium oxylepis fo. marianum Ruíz Torre \& Ruíz Cast.

Teucrium oxylepis subsp. oxylepis Font Quer

Teucrium polium $\mathrm{L}$.

Teucrium valentinum Schreb.

Teucrium pseudoscorodonia Desf.

Teucrium salviastrum Schreb.

Teucrium scorodonia $\mathrm{L}$.

Thymus broussonettii Boiss.

Thymus capitatus (L.) Hoffmanns. \& Link

Thymus cilicicus Boiss. \& Balansa

Thymus funkii Coss.

Thymus longicaulis C. Presl

Thymus mastichina L.

Thymus orospedanus Huguet del Villar

Thymus riatarum Humbert \& Maire

Thymus saturejoides Coss.

Thymus serpyllum $\mathrm{L}$.

Thymus vulgaris $\mathrm{L}$.

Thymus $x$ citriodorus Schreb.

Thymus zygis L. caryophyllene oxide

thymol, caryophyllene oxide

caryophyllene oxide

caryophyllene oxide

thymol, caryophyllene oxide

caryophyllene oxide

pulegone, thymol

thymol

pulegone, thymol, menthone

pulegone, menthone

pulegone, menthone

thymol

thymol, caryophyllene oxide

thymol, caryophyllene oxide

pulegone

pulegone, menthone

thymol

thymol

caryophyllene oxide

menthone

caryophyllene oxide

thymol

thymol, anethole

caryophyllene oxide

thymol

thymol, caryophyllene oxide, anethole

thymol, caryophyllene oxide, anethole

thymol

thymol, caryophyllene oxide, anethole

thymol, caryophyllene oxide, anethole

thymol

thymol

pulegone

thymol

thymol

thymol

thymol

thymol

eugenol, thymol, caryophyllene oxide eugenol, caryophyllene oxide eugenol, linalool, thymol thymol, caryophyllene oxide thymol, caryophyllene oxide

thymol

thymol, caryophyllene oxide

thymol

thymol

eugenol, thymol, menthone, anethole

thymol

thymol 


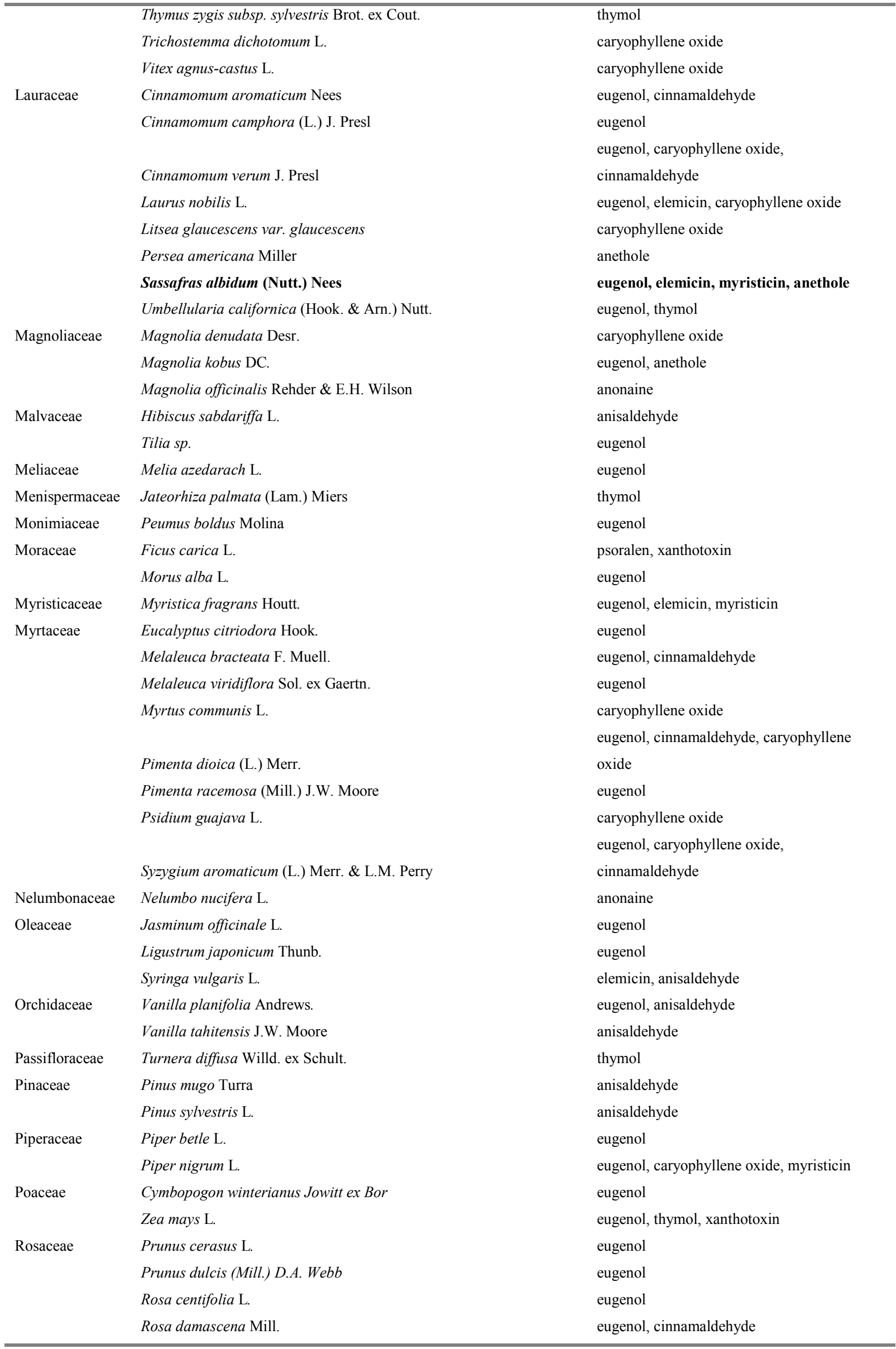




\begin{tabular}{|c|c|c|}
\hline & Rosa gallica L. & eugenol \\
\hline Rubiaceae & Coffea arabica $\mathrm{L}$. & eugenol \\
\hline \multirow[t]{13}{*}{ Rutaceae } & Aegle marmelos Correa & psoralen \\
\hline & Agathosma betulina (P.J. Bergius) Pillans & pulegone \\
\hline & Barosma betulina Bartl. \& H.L. Wendl. & pulegone \\
\hline & Boronia megastigma Nees ex Bartl. & eugenol \\
\hline & Casimiroa edulis Llave \& Lex. & isopimpinellin \\
\hline & Citrus aurantiifolia (Christm.) Swingle & isopimpinellin \\
\hline & Citrus aurantium $\mathrm{L}$. & thymol \\
\hline & Citrus limon (L.) Osbeck & thymol, caryophyllene oxide, isopimpinellin \\
\hline & Citrus reticulata Blanco & thymol \\
\hline & Dictamnus albus L. & psoralen, xanthotoxin \\
\hline & Limonia acidissima $\mathrm{L}$. & isopimpinellin, psoralen \\
\hline & Ruta graveolens L. & isopimpinellin, psoralen, xanthotoxin \\
\hline & Skimmia arborescens T. Anderson Ex Gamble & isopimpinellin \\
\hline Santalaceae & Santalum album $\mathrm{L}$. & eugenol \\
\hline Schisandraceae & Illicium verum Hook. f. & anisaldehyde \\
\hline \multirow[t]{8}{*}{ Solanaceae } & Capsicum annuum $\mathrm{L}$. & eugenol, pulegone \\
\hline & Capsicum frutescens $\mathrm{L}$. & pulegone \\
\hline & Lycopersicon esculentum Mill. & eugenol, cinnamaldehyde, tomatine \\
\hline & Nicotiana tabacum L. & eugenol \\
\hline & Solanum carolinense L. & solamargine \\
\hline & Solanum dulcamara $\mathrm{L}$. & solamargine \\
\hline & Solanum melongena $\mathrm{L}$. & solamargine \\
\hline & Solanum nigrum L. & solamargine \\
\hline Theaceae & Camellia sinensis (L.) Kuntze & eugenol, thymol \\
\hline \multirow[t]{3}{*}{ Verbenaceae } & Aloysia citriodora Palau & caryophyllene oxide \\
\hline & Lantana camara $\mathrm{L}$. & eugenol \\
\hline & Lippia graveolens Kunth & thymol \\
\hline Violaceae & Viola odorata L. & eugenol \\
\hline Winteraceae & Drimys winteri J.R. Forst. \& G. Forst. & eugenol \\
\hline \multirow[t]{3}{*}{ Zingiberaceae } & Alpinia galanga (L.) Willd. & eugenol, caryophyllene oxide \\
\hline & Alpinia officinarum Hance & eugenol \\
\hline & Curcuma longa $\mathrm{L}$. & eugenol \\
\hline
\end{tabular}

Chemical compounds of the Lamiaceae family appear to be the most promising for management of leaf cutting ants (Figures 3 and 5). Indeed, several authors cited this family as one of the dominant families among aromatic plants with antifungal and insecticidal activities (Vokou et al. 1998; Kokkini et al 1988; González et al. 2011). The specific advantages of these plants are their secondary metabolites, such as terpenoids and phenolics, which are known to have strong plant chemical defense activity against insects, bacteria and fungi (Karamanoli et al. 2000). Other studies revealed that species of the Apiaceae family possess chemical compounds with diverse biological activities such as apoptosis inducers, antibacterial, antifungal, phytotoxic activity, and cyclooxygenase inhibitory (Meepagala et al. 2005) that may potentially be used for pest management.

Phenolic compounds can be divided into simple phenols, phenolic acids, quinones, flavones, flavonoids, flavonols, tannins and coumarins (Murphy Cowan 1999). The sites and number of hydroxyl groups on the phenolic compounds are thought to be related to their relative toxicity to fungi (Geissman 1963). Terpenoids occur as diterpenes, triterpenes, tetraterpenes, hemiterpenes and sesquiterpenes (Murphy Cowan 1999). Their mechanism of action against fungi is speculated to involve membrane disruption by the lipophilic compounds (Mendoza et al. 1997). Alkaloids are heterocyclic nitrogen compounds. Their antimicrobial mechanism of action is attributed to their ability to intercalate with DNA (Phillipson an O’Neil 1987). 
Phytochemicals from plants include substances that are potentially useful as insect control. While synthetic insecticides have neurotoxic mode of action and promoted the rapid developpement of cross-resistance in insect population; phytochemical insecticides have emphasized non-neurotoxic modes-of-action such as antifeedant action, inhibition of molting, growth reduction, loss of fecundity, respiratory inhibition, etc. Standardized plant extracts containing a mixture of active phytochemicals should reduce the rate of evolution of conventional resistance compared to the selection pressure exerted by single pure toxin (Arnason et al. 1993). Studies, on relationships between chemicals structure and activity in phytochemical compounds, revealed that one important factor of insecticidal activities is related to the number of hydroxyl functions available compared to the degree of polymerization of the molecule (Regnault-Roger et al. 2003).

A choice must also be made for extraction type and solvent used. The choice would be influenced most by the degradability of total plant extract obtained. Water seems to be the best solvent. All the molecules with hydroxyl or glycated functions were water-soluble. Alkaloids were also miscible in aqueous solvents, but which have acid $\mathrm{pH}$. Being naturally evolved ingredients such as plants and water have at least an advantage over synthetic molecules in term of ecological suitability. Their development as succesfull pest control products can also be economically feasible, especially if the source materials and solvent are available in abundance or with lower price (e.g., common plants with a wide distribution and water which is the least expensive extraction solvent).

Among methods for controlling Attini ants, the use of toxic baits is probably the most efficient. These baits should contain an active ingredient used on adult ant workers (Camargo et al. 2006). Thus, with our prespective to find efficient natural method to control leaf-cutting ants, the ingestion baits containing water plant extracts with an effective fungicidal and insecticidal action could be tested and then used.

\section{Conclusion}

In this study based on exhaustive literature review, we have identified twenty chemical compounds and seventeen plant species from the Lamiaceae and Apiaceae families with potential to achieve efficient pest management of leaf cutting ants. These seventeen species include four to seven chemical compounds with both insecticidal and fungicidal activities. Further biological bioassays will be conducted to evaluate the experimental effect of extracts from the plant species extracts identified from this revew on Attini ants and their symbiotic fungus.

\section{Acknowledgements}

The authors thank CEREGMIA and his director Fred Celimène for financial support of I. Boulogne. Special thanks to Patrice Champoiseau for his suggestions.

\section{References}

Adfa M, Yoshimura T, Komura K, Koketsu M (2010) Antitermite Activities of Coumarin Derivatives and Scopoletin from Protium javanicum Burm. f. J Chem Ecol 36:720-726.

Agnihotri VK, ElSohly HN, Khan SI, Jacob MR, Joshi VC, Smillie T, Khan IA, Walker LA (2008) Constituents of Nelumbo nucifera leaves and their antimalarial and antifungal activity. Phytochem Lett 1:89-93.

Akhtar Y, Isman MB (2003) Larval Exposure to Oviposition Deterrents Alters Subsequent Oviposition Behavior in Generalist, Trichoplusia $n i$ and Specialist, Plutella xylostella Moths. J Chem Ecol 29:1853-1870.

Akhtar Y, Isman MB (2004) Generalization of a Habituated Feeding Deterrent Response to Unrelated Antifeedants Following Prolonged Exposure in a Generalist Herbivore, Trichoplusia ni. J Chem Ecol 30:1349-1362. 
Al-Barwani FM, Eltayeb EA (2004) Antifungal compounds from induced Conium maculatum L. plants. Biochem Syst Ecol 32:1097-1108.

Ameziane N, Boubaker H, Boudyach H, Msanda F, Jilal A, Ait Benaoumar A (2007) Antifungal activity of Moroccan plants against citrus fruit pathogens. Agron Sustain Dev 27:273-

277.

Amicbase (2010) Collections of Antimicrobial Data of Drugs, Natural Compounds and Essential Oils. http://www.reviewscience.com/Index.html. Acessed 11 October 2010.

Antunes EC, Guedes RNC, Della Lucia TMC, Serrão JE (2000) Sub-lethal effects of abamectin suppressing colonies of the leaf-cutting ant Acromyrmex subterraneus subterraneus.

Pest Manag Sci 56:1059-1064.

Arnason JT, MacKinnon S, Durst A, Philogène BJR, Hasbun C, Sanchez P, Poveda L, San Roman L, Isman MB, Satasook C, Towers GHN, Wiriyachitra P, MacLaughlin JL (1993) Insecticides in tropical plants with non-neurotoxic modes of action. In: Phytochemical Potential of Tropical plants. Recent advances in phytochemistry 27. Plenum Press, New York.

Asthana A, McCloud ES, Berenbaum MR, Tuveson RW (1993) Phototoxicity of Citrus jambhiri to fungi under enhanced UV-B radiation: Role of furanocoumarins. J Chem Ecol 19: 2813-2830.

Bacci Jr M, Solomon SE, Mueller UG, Martins VG, Carvalho AOR, Vieira LGE, Silva-Pinhati ACO (2009) Phylogeny of leafcutter ants in the genus Atta fabricius (Formicidae: Attini) based on mitochondrial and nuclear DNA sequences. Mol Phylogenet Evol 51:427-437.

Bafi-Yeboa NFA, Arnason JT, Baker J, Smith ML (2005) Antifungal constituents of Northern prickly ash, Zanthoxylum americanum Mill. Phytomedicine 12:370-377.

Benckiser G (2010) Ants and sustainable agriculture. A review. Agronomy for Sustainable Development 30:191-199.

Bigi MFMA, Torkomian VLV, de Groote STCS, Hebling MJA, Bueno OC, Pagnocca FC, Fernandes JB, Vieira PC, da Silva MFGF (2004) Activity of Ricinus communis (Euphorbiaceae) and ricinine against the leaf-cutting ant Atta sexdens rubropilosa

(Hymenoptera:Formicidae) and the symbiotic fungus Leucoagaricus gongylophorus. Pest Manag Sci 60:933-938.

Bloem KA, Kelley KC, Duffey SS (1989) Differential effect of tomatine and its alleviation by cholesterol on larval growth and efficiency of food utilization in Heliothis zea and Spodoptera exigua. J Chem Ecol 15:387-398.

Borg-Karlson A-K, Nordlander G, Mudalige A, Nordenhem H, Unelius CR, (2006) Antifeedants in the Feces of the Pine Weevil Hylobius abietis: Identification and Biological Activity. J Chem Ecol 32:943-957.

Boulogne I (2011) Évaluation du potentiel insecticide et antifongique sur Acromyrmex octospinosus (Reich) d'une sélection de plantes à usages ethnopharmacologiques TRAMIL. Thèse de doctorat Université des Antilles et de la Guyane.

Boulogne I, Germosén-Robineau L, Ozier-Lafontaine H, Jacoby-Koaly C, Aurela L, LorangerMerciris G (2011) Acromyrmex octospinosus (Hymenoptera: Formicidae) management. Part 1. Effects of TRAMIL's insecticidal plant extracts. Pest Manag Sci 68 (2): 313-320. 
Braga PC, Culici M, Alfieri M, Dal Sasso M (2008) Thymol inhibits Candida albicans biofilm formation and mature biofilm. Int J Antimicrob Ag 31:472-477.

Bruneton J (1999) Pharmacognosie. Phytochimie, plantes médicinales. 3rd edn. Tec \& Doc, Paris.

Camargo RS, Forti LC, Lopes JFS, Nagamoto NS (2006) Studies on leafcutting ants, Acromyrmex spp. (Formicidae, Attini): behavior, reproduction andcontrol. Recent Res Dev Entomol 5:6182 .

Chang KS, Ahn YJ (2002) Fumigant activity of (E)-anethole identified in Illicium verum fruit against Blattella germanica. Pest Manag Sci 58:161-166.

Cheng SS, Liu JY, Chang EH, Chang ST (2008) Antifungal activity of cinnamaldehyde and eugenol congeners against wood-rot fungi. Bioresource Technol 99:5145-5149.

Cheng SS, Wu CL, Chang HT, Kao YT, Chang ST (2004) Antitermitic and Antifungal Activities of Essential Oil of Calocedrus formosana Leaf and Its Composition. J Chem Ecol 30:1957-1967.

Daferera DJ, Ziogas BN, Polissiou MG (2003) The effectiveness of plant essential oils on the growth of Botrytis cinerea, Fusarium sp. and Clavibacter michiganensis subsp. michiganensis. Crop Prot 22:39-44.

Dambolena JS, López AG, Cánepa MC, Theumer MG, Zygadlo JA, Rubinstein HR (2008) Inhibitory effect of cyclic terpenes (limonene, menthol, menthone and thymol) on Fusarium verticillioides MRC 826 growth and fumonisin B1 biosynthesis. Toxicon 51:37-44.

Dancewicz K, Gabrys B, Dams I, Wawrzeńczyk C (2008) Enantiospecific Effect of Pulegone and Pulegone-Derived Lactones on Myzus persicae (Sulz.) Settling and Feeding. J Chem Ecol 34:530-538.

De Paula VF, De Barbosa LCA, Demuner AJ, Pilo-Veloso D, Picanco MC (2000) Synthesis and insecticidal activity of new amide derivatives of piperine. Pest Manag Sci 56:168-174.

Di X, Liu Y, Liu Y, Yu X, Xiao H, Tian X, Gao R (2010) Synthesis and insecticidal activities of pyridine ring derivatives of podophyllotoxin. Pestic Biochem Phys 89:81-87.

Diawara MM, Trumble JT, White KK, Carson WG,Martinez LA (1993) Toxicity of linear furanocoumarins to Spodoptera exigua: Evidence for antagonistic interactions. J Chem Ecol 19: $2473-2484$.

Duke JA (1992) Handbook of phytochemical constituents of GRAS herbs and other economic plants. FL. CRC Press, Boca Raton.

Duke JA (2010) Dr. Duke's Phytochemical and Ethnobotanical Databases. http://www.arsgrin.gov/duke. Accessed 22 September 2010.

Dussourd DE (2003) Chemical Stimulants of Leaf-Trenching by Cabbage Loopers: Natural Products, Neurotransmitters, Insecticides, and Drugs. J Chem Ecol 29:2023-2047.

Erthal Jr M, Peres Silva C, Samuels RI (2004) Digestive enzymes of leaf-cutting ants, Acromyrmex subterraneus (Hymenoptera: Formicidae:Attini): distribution in the gut of adult workers and partial characterization. J Insect Physiol 50:881-891. 
Ferhout H, Bohatier J, Guillot J, Chalchat JC (1999) Antifungal activity of selected essential oils, cinnamaldehyde and carvacrol against Malassezia furfur and Candida albicans. J Essent Oil Res 11:119-129.

Fewell AM, Roddick JG, Weissenberq M (1994) Interaction between the glycoalkaloids solasonine and solamargine in relation to inhibition of fungal growth. Phytochemistry 37:10071011.

Figgitt DP, Denyer SP, Dewick PM, Jackson DE, Williams P (1989) Topoisomerase II: A potential target for novel antifungal agents. Biochem Bioph Res Co 160:257-262.

Forti LC, Pretto DR, Nagamoto NS, Padovani CR, Camargo RS, Andrade APP (2007) Dispersal Of the Delayed Action Insecticide Sulfluramid In Colonies of the Leaf-Cutting Ant Atta sexdens rubropilosa (Hymenoptera : Formicidae ). Sociobiology 50 (3):1-15.

Fowler HG (1978) Foraging Trails of Leaf-Cutting Ants. Journal of the New York Entomological Society 86:132-136.

Gallardo F, Boethel DJ, Fuxa JR, Richter A (1990) Susceptibility of Heliothis zea (Boddie) larvae to Nomuraea rileyi (Farlow) Samson Effects of $\alpha$-tomatine at the third trophic level. J Chem Ecol 16:1751-1759.

Galván IJ, Mir-Rashed N, Jessulat M, Atanya M, Golshani A, Durst T, Petit P, Amiguet VT, Boekhout T, Summerbell R, Cruz I, Arnason JT, Smith ML (2008) Antifungal and antioxidant activities of the phytomedicine pipsissewa, Chimaphila umbellata. Phytochemistry 69:738-746.

Ganapaty S, Thomas PS, Fotso S, Laatsch H (2004) Antitermitic quinones from Diospyros sylvatica. Phytochemistry 65:1265-1271.

Gao R, Gao CXT, Yu X, Di X, Xiao H, Zhang X (2004) Insecticidal activity of deoxypodophyllotoxin, isolated from Juniperus sabina L, and related lignans against larvae of Pieris rapae L. Pest Manag Sci 60:1131-1136.

Gawde AJ, Cantrell CL, Zheljazkov VD (2009) Dual extraction of essential oil and podophyllotoxin from Juniperus virginiana. Ind Crop Prod 30:276-280.

Gayoso CW, Lima EO, Oliveira VT, Pereira FO, Souza EL, Lima IO, Navarro DF (2005) Sensitivity of fungi isolated from onychomycosis to Eugenia cariophyllata essential oil and eugenol. Fitoterapia 76:247-249.

Geissman TA (1963) Flavonoid compounds, tannins, lignins and related compounds. In: M Florkin and EH Stotz (ed) Pyrrole pigments, isoprenoid compounds and phenolic plant constituents 9. Elsevier, New York.

González JOW, Gutiérrez MM, Murray AP, Ferrero AA (2011) Composition and biological activity of essential oils from Labiatae against Nezara viridula (Hemiptera: Pentatomidae) soybean pest. Pest Manag Sci 67: 948-955.

Güntner C, Vázquez A, González G, Usubillaga A, Ferreira F, Moyna P (2000) Effect of Solanum Glycoalkaloids on Potato Aphid, Macrosiphum euphorbiae: Part $\quad$ II. J Chem Ecol 26:1113- $\quad 1121$.

Hadaček F, Müller C, Werner A, Greger H, Proksch P (1994) Analysis, isolation and insecticidal activity of linear furanocoumarins and other coumarin derivatives from Peucedanum (Apiaceae: Apioideae). J Chem Ecol 20:2035-2054. 
Hall CA, Hobby T, Cipollini M (2006) Efficacy and Mechanisms of $\alpha$-Solasonine and $\alpha$-SolamargineInduced Cytolysis on Two Strains of Trypanosoma cruzi. J Chem Ecol 32:2405-2416.

Harper SH, Potter C, Gillham EM (1947) Annona species as insecticides. Ann Appl Biol 34:104-112.

Huang Y, Ho S-H, Lee H-C, Yap Y-L (2002) Insecticidal properties of eugenol, isoeugenol and methyleugenol and their effects on nutrition of Sitophilus zeamais Motsch. (Coleoptera: Curculionidae) and Tribolium castaneum (Herbst) (Coleoptera: Tenebrionidae). Journal of Stored Products Research 38:403-412.

Hubert J, Munzbergova Z, Santino A (2008) Plant volatile aldehydes as natural insecticides against stored-product beetles. Pest Manag Sci 64:57-64.

Islam R, Islam Khan R, Al-Reza SM, Jeong YT, Song CH, Khalequzzaman M (2009) Chemical composition and insecticidal properties of Cinnamomum aromaticum (Nees) essential

oil against the stored product beetle Callosobruchus maculatus (F.). J Sci Food Agr 89:12411246.

Ito SI, Ihara T, Tamura H, Tanaka S, Ikeda T, Kajihara H, Dissanayake C, Abdel-Motaal FF, El-Sayed MA (2007) $\alpha$-Tomatine, the major saponin in tomato, induces programmed cell death mediated by reactive oxygen species in the fungal pathogen Fusarium oxysporum. FEBS Lett 581:3217-3222.

Javaid I, Dadson RB, Hashem FM, Joshi J (2006) Antibiosis of forage soybean as an ecological alternative for the control of corn earworm, Agron Sustain Dev 26:55-59.

Jianhua W, Hai W (2001) Antifungal susceptibility analysis of berberine, baicalin, eugenol and curcumin on Candida albicans. J Med Colleges of PLA 24:142-147.

Karamanoli K, Vokou D, Menkissoglu U, Constantinidou H-I (2000) Bacterial Colonization of Phyllosphere of Mediterranean Aromatic Plants. J Chem Ecol 26:2035-2048.

Karapinar M (1990) Inhibitory effects of anethole and eugenol on the growth and toxin production of Aspergillus parasiticus. Int J Food Microbiol 10:193-199.

Keszei A, Hassan Y, Foley WJ (2010) A Biochemical Interpretation of Terpene Chemotypes in Melaleuca alternifolia. J Chem Ecol 36:652-661.

Kokkini S, Karagiannakidou V, Hanlidou E, Vokou D (1988) Geographical and altitudinal distribution of the Lamiaceae in Greece. Phyton 28:215-228.

Kordali S, Cakir A, Ozer H, Cakmakci R, Kesdek M, Mete E (2008) Antifungal, phytotoxic and insecticidal properties of essential oil isolated from Turkish Origanum acutidens and its three components, carvacrol, thymol and p-cymene. Bioresource Technol 99:8788-8795.

Kotkar HM, Mendki PS, Sadan SVGS, Jha SR, Upasani SM, Maheshwari VL (2002) Antimicrobial and pesticidal activity of partially purified flavonoids of Annona squamosa. Pest Manag Sci 58:33-37.

Kubo I (1993) Insect control agents from tropical plants. In: Phytochemical Potential of Tropical plants. Recent advances in phytochemistry 27. Plenum Press, New York.

Lee S, Peterson CJ, Coats JR (2003) Fumigation toxicity of monoterpenoids to several stored product insects. J Stored Prod Res 39:77-85. 
Lee SE, Lee BH, Choi WS, Park BS, Kim JG, Campbell BC (2001) Fumigant toxicity of volatile natural products from Korean spices and medicinal plants towards the rice weevil, Sitophilus oryzae (L). Pest Manag Sci 57:548-553.

Lichtfouse E, Navarrete M, Debaeke P, Souchère V, Alberola C, Ménassieu J (2009) Agronomy for sustainable agriculture. A review. Agron Sustain Dev 29:1-6.

Lichtfouse E, Schwarzbauer J, Robert D (2011). Social chemistry. Environ Chem Lett DOI 10.1007/s10311-011-0333-3.

Martínez-Romero D, Serrano M, Bailén G, Guillén F, Zapata PJ, Valverde JM, Castillo S, Fuentes M, Valero D (2008) The use of a natural fungicide as an alternative to preharvest synthetic fungicide treatments to control lettuce deterioration during postharvest storage. Postharvest Biol Tec 47:54-60.

Maya JD, Cassels BK, Iturriaga-Vásquez P, Ferreira J, Faúndez M, Galanti N, Ferreira A, Morello A (2007) Mode of action of natural and synthetic drugs against Trypanosoma cruzi and their interaction with the mammalian host. Comparative Biochemistry and Physiology - Part A: Molecular \& Integrative Physiology 146:601-620.

Meepagala KM, Sturtz G, Wedge DE, Schrader KK, Duke SO (2005) Phytotoxic and Antifungal Compounds from Two Apiaceae Species, Lomatium californicum and Ligusticum hultenii, Rich Sources of Z-ligustilide and Apiol, Respectively. J Chem Ecol 31:1567-1578.

Mendoza L, Wilkens M, Urzua A (1997) Antimicrobial study of the resinous exudates and of diterpenoids and flavonoids isolated from some Chilean Pseudognaphalium

(Asteraceae). J Ethnopharmacol 58:85-88.

Mikheyev AS (2008) History, genetics and pathology of a leaf-cutting ant introduction: a case study of the Guadeloupe invasion. Biol Invasions 10:467473.

Mishra BB, Singh DD, Kishore N, Tiwari VK, Tripathi V (2010) Antifungal constituents isolated from the seeds of Aegle marmelos. Phytochemistry 71:230-234.

Mitchell BK, Harrison GD (1985) Effects of Solanum glycoalkaloids on chemosensilla in the Colorado potato beetle. J Chem Ecol 11:73-83.

Monsálvez M, Zapata N, Vargas M, Berti M, Bittner M, Hernández V (2010) Antifungal effects of nhexane extract and essential oil of Drimys winteri bark against Take-All disease. Ind Crop Prod 31:239-244.

Multigner L, Ndong JR, Giusti A, Romana M, Delacroix-Maillard H, Cordier S, Jegou B, Thome JP, Blanchet P (2010) Chlordecone Exposure and Risk of Prostate Cancer. J Clin Oncol 28:34573462 .

Murphy Cowan M (1999) Plant Products as Antimicrobial Agents. Clinical Microbiology Reviews 12:564-582.

Nenaah G (2010) Individual and synergistic toxicity of solanaceous glycoalkaloids against two coleopteran stored-product insects. J Pest Sci doi:10.1007/s103400100329-y.

Neri F, Mari M, Brigati S (2005) Control of Penicillium expansum by plant volatile compounds. Plant Pathol 55:100-105. 
Ngoh SP, Choo LEW, Pang FY ,Huang Y, Kini MR, Ho SH (1998) Insecticidal and repellent properties of nine volatile constituents of essential oils against the American cockroach, Periplaneta americana (L.). Pesticide Science 54:261-268.

Osei-Safo D, Addae-Mensah I, Garneau FX, Koumaglo HK (2010) A comparative study of the antimicrobial activity of the leaf essential oils of chemo-varieties of Clausena anisata (Willd.) Hook. f. ex Benth. Ind Crop Prod 32:634-638.

Pagnocca FC, Ribeiro SB, Torkomian VLV, Hebling MJA, Bueno OC, da Silva OA, Fernandes JB, Vieira PC, da Silva MFGF, Ferreira AG (1996) Toxicity of lignans to symbiotic fungus of leaf-cutting ants. J Chem Ecol 22:1325-1330.

Pagnocca FC, Victor SR, Bueno FC, Crisostomo FR, Castral TC, Fernandes JB, Correa AG, Bueno OC, Bacci M, Hebling MJA, Vieira PC, da Silva MFGF (2006) Synthetic amides toxic

to the leaf-cutting ant Atta sexdens rubropilosa L. and its symbiotic fungus. Agr Forest Entomol $8: 17-23$.

Paneru RB, le Patourel GNJ, Kennedy SH (1997) Toxicity of Acorus calamus rhizome powder from Eastern Nepal to Sitophilus granaries (L.) and Sitophilus oryzae (L.) (Coleoptera, Curculionidae). Crop Prot 16:759-763.

Paoletti MG, Pimentel D (2000) Environmental Risks of Pesticides Versus Genetic Engineering for Agricultural Pest Control. J Agr Environ Ethic 12:279-303.

Paoletti MG, Pimentel D (2000) Environmental Risks of Pesticides Versus Genetic Engineering for Agricultural Pest Control. J Agr Environ Ethic 12:279-303.

Park IK, Choi KS, Kim DH, Choi IH, Kim LS, Bak WC, Choi JW, Shin SC (2006) Fumigant activity of plant essential oils and components from horseradish (Armoracia rusticana), anise (Pimpinella anisum) and garlic (Allium sativum) oils against Lycoriella ingenua (Diptera: Sciaridae). Pest Manag Sci 62:723-728.

Petit P (2004) Contribution à l'étude chimique de Lonchocarpus floribundus Bentham et de Lonchocarpus chrysophyllus Kleinhoonte. Synthèse de derives soufrés à partir d'éthers phénoliques naturels. Thèse de doctorat Université des Antilles et de la Guyane.

Phillipson JD, O'Neill MJ (1987) New leads to the treatment of protozoal infections based on natural product molecules. Acta Pharm Nord 1:131-144.

Picman AK (1984) Antifungal activity of sesquiterpene lactones. Biochem Syst Ecol 12:13-18.

Qin W, Huang S, Li C, Chen S, Peng Z (2010) Biological activity of the essential oil from the leaves of Piper sarmentosum Roxb. (Piperaceae) and its chemical constituents on Brontispa longissima (Gestro) (Coleoptera: Hispidae). Pestic Biochem Phys 96:132-139.

Rakotonirainy MS, Lavedrine B (2005) Screening for antifungal activity of essential oils and related compounds to control the biocontamination in libraries and archives storage areas. Int Biodeter Biodegr 55:141-147.

Rattan RS (2010) Mechanism of action of insecticidal secondary metabolites of plant origin. Crop Prot 29:913-920.

Regnault-Roger C, Philogene BJR, Vincent C (2003) Biopesticides d'origine végétale. Tec\&Doc Lavoisier, Paris. 
Ribeiro KAL, Monteiro de Carvalho C, Molina MT, Pereira Lima E, López-Montero E, Rui M Reys J, Farias de Oliveira MB, Ventura Pinto A, Santana AEG, Goulart MOF (2009) Activities of naphthoquinones against Aedes aegypti (Linnaeus, 1762) (Diptera: Culicidae), vector of dengue and Biomphalaria glabrata (Say, 1818), intermediate host of Schistosoma mansoni. Acta Trop 111:44-50.

Salvatore A, Borkosky S, Willink E, Bardón (2004) A Toxic Effects of Lemon Peel Constituents on Ceratitis capitata. J Chem Ecol 30:323-333.

Shaaya E, Ravid U, Paster N, Juven B, Zisman U, Pissarev V (1991) Fumigant toxicity of essential oils against four major stored-product insects. J Chem Ecol 17:499-504.

Silva-Pinhati ACO, Bacci Jr M, Hinkle G, Sogin ML, Pagnocca FC, Martins VG, Bueno OC, Hebling MJA (2004) Low variation in ribosomal DNA and internal transcribed spacers of the symbiotic fungi of leaf-cutting ants (Attini: Formicidae). Braz J Med Biol Res 37:1463-1472.

Silva-Pinhati ACO, Bacci JrM, Siqueira CG, Silva A, Pagnocca FC, Bueno OC, Hebling MJA (2005) Isolation and Maintenance of Symbiotic Fungi of Ants in the Tribe Attini (Hymenoptera: Formicidae). Neotrop Entomol 34:1-5.

Silva A, Bacci M Jr, Pagnocca FC, Bueno OC, Hebling MJA (2006) Starch metabolism in Leucoagaricus gongylophorus, the symbiotic fungus of leaf-cutting ants. Microbiol Res 161:299-303.

Simas NK, Ferrari SF, Pereira SN, Leitão GG (2001) Chemical Ecological Characteristics of Herbivory of Siparuna guianensis Seeds by Buffy-Headed Marmosets (Callithrix flaviceps) in the Atlantic Forest of Southeastern Brazil. J Chem Ecol 27:93-107.

Sreelatha T, Hymavathi A, Murthy JM, Rani PU, Rao JM, Babu KS (2010) Bioactivity-guided isolation of mosquitocidal constituents from the rhizomes of Plumbago capensis Thunb. Bioorgan Med Chem Lett 20:2974-2977.

Staneva J, Denkova P, Todorova M, Evstatieva L (2010) Quantitative analysis of sesquiterpene lactones in extract of Arnica Montana L. by ${ }^{1} \mathrm{H}$ NMR spectroscopy. J Pharmaceut Biomed doi:10.1016/j.jpba.2010.08.018.

Tavares AC, Gonçalves MJ, Cavaleiro C, Cruz MT, Lopes MC, Canhoto J, Salgueiro LR (2008) Essential oil of Daucus carota subsp. halophilus: Composition, antifungal activity and cytotoxicity. J Ethnopharmacol 119:129-134.

Tellez M, Estell R, Fredrickson E, Powell J, Wedge D, Schrader K, Kobaisy M (2001) Extracts of Flourensia cernua (L): Volatile constituents and antifungal, antialgal, and antitermite bioactivities. J Chem Ecol 27:2263-2273.

Traboulsi AF, Taoubi K, El-Haj S, Bessiere JM, Rammal S (2002) Insecticidal properties of essential plant oils against the mosquito Culex pipiens molestus (Diptera: Culicidae). Pest Manag Sci $58: 491-495$.

Treyvaud Amiguet V, Petit P, Ta CA, Nuñez R, Sánchez-Vindas P, Poveda Alvarez L, Smith ML, Arnason JT, Durst T (2006) Phytochemistry and Antifungal Properties of the Newly Discovered Tree Pleodendron costaricense. J Nat Prod 69:1005-1009.

Tropicos (2010) Tropicos.org. Missouri Botanical Garden. http://www.tropicos.org. Accessed 27 September 2010. 
Vagionas K, Ngassapa O, Runyoro D, Graikou K, Gortzi O, Chinou I (2007) Chemical analysis of edible aromatic plants growing in Tanzania. Food Chem 105:1711-1717.

Vázquez BI, Fente C, Franco CM, Vázquez MJ, Cepeda (2001) A Inhibitory effects of eugenol and thymol on Penicillium citrinum strains in culture media and cheese. Int $\mathrm{J}$ Food Microbiol 67:157-163.

Vencl FV, Morton TC, Mumma RO, Schultz JC (1999) Shield Defense of a Larval Tortoise Beetle. J Chem Ecol 25:549-566.

Vokou D, Tziolas M, Bailey SER (1998) Essential-Oil-Mediated Interactions Between Oregano Plants and Helicidae Grazers. J Chem Ecol 24:1187-1202.

Waliwitiya R, Kennedy CJ, Lowenberger CA (2009) Larvicidal and oviposition-altering activity of monoterpenoids, trans-anithole and rosemary oil to the yellow fever mosquito Aedes aegypti (Diptera: Culicidae). Pest Manag Sci 65:241-248.

$\mathrm{Xu} \mathrm{H}, \mathrm{He}$ XQ (2010) Natural products-based insecticidal agents 6. Design, semisynthesis, and insecticidal activity of novel monomethyl phthalate derivatives of podophyllotoxin against Mythimna separata Walker in vivo. Bioorgan Med Chem Lett 20: 4503-4506.

Xuan TD, Elzaawely AA, Deba F, Fukuta M, Tawata S (2006) Mimosine in Leucaena as a potent bioherbicide, Agron Sustain Dev 26:89-97.

Zhu BCR, Henderson G, Chen F, Fei H, Laine RA (2001) Evaluation of Vetiver Oil and Seven InsectActive Essential Oils Against the Formosan Subterranean Termite. J Chem Ecol 27:16171625.

Zhu BCR, Henderson G, Sauer AM, Yu Y, Crowe W, Laine RA (2003) Structure-Activity of Valencenoid Derivatives and Their Repellence to the Formosan Subterranean Termite. J Chem Ecol 29:2695-2701. 